\title{
Hybrid Dispersive Optical Solitons in Nonlinear Cubic-Quintic-Septic Schrödinger Equation
}

\author{
Clovis Taki Djeumen Tchaho1,2*, Hugues Martial Omanda1,3, Gaston N'tchayi Mbourou", \\ Jean Roger Bogning5, Timoléon Crépin Kofané6,7
}

\author{
${ }^{1}$ African Centre for Advanced Studies, Yaoundé, Cameroon \\ ${ }^{2}$ Lycée Technique Fulbert Bongotha, Moanda, Gabon \\ ${ }^{3}$ Laboratoire Pluridisciplinaire des Sciences, Ecole Normale Supérieure, Libreville, Gabon \\ ${ }^{4}$ Laboratoire de Mécanique des Matériaux, Ecole Polytechnique, Université des Sciences et Techniques de Masuku, Franceville, \\ Gabon \\ ${ }^{5}$ Department of Physics, Higher Teacher Training College, University of Bamenda, Bamenda, Cameroon \\ ${ }^{6}$ Department of Physics, Faculty of Science, University of Yaoundé I, Yaoundé, Cameroon \\ ${ }^{7}$ Centre d'Excellence Africain en Technologie de l'Information et de la Télécommunication, University of Yaoundé I, Yaoundé, \\ Cameroon \\ Email: *djeumenclovis@gmail.com, ^djeumentchaho@acas-yde.org
}

How to cite this paper: Tchaho, C.T.D., Omanda, H.M., Mbourou, G.N., Bogning, J.R. and Kofané, T.C. (2021) Hybrid Dispersive Optical Solitons in Nonlinear CubicQuintic-Septic Schrödinger Equation. Optics and Photonics Journal, 11, 23-49. https://doi.org/10.4236/opj.2021.112003

Received: January 11, 2021

Accepted: February 23, 2021

Published: February 26, 2021

Copyright $\odot 2021$ by author(s) and Scientific Research Publishing Inc. This work is licensed under the Creative Commons Attribution International License (CC BY 4.0).

http://creativecommons.org/licenses/by/4.0/

\begin{abstract}
Certain hybrid prototypes of dispersive optical solitons that we are looking for can correspond to new or future behaviors, observable or not, developed or will be developed by optical media that present the cubic-quintic-septic law coupled, with strong dispersions. The equation considered for this purpose is that of non-linear Schrödinger. The solutions are obtained using the Bogning-Djeumen Tchaho-Kofané method extended to the new implicit Bogning' functions. Some of the obtained solutions show that their existence is due only to the Kerr law nonlinearity presence. Graphical representations plotted have confirmed the hybrid and multi-form character of the obtained dispersive optical solitons. We believe that a good understanding of the hybrid dispersive optical solitons highlighted in the context of this work allows to grasp the physical description of systems whose dynamics are governed by nonlinear Schrödinger equation as studied in this work, allowing thereby a relevant improvement of complex problems encountered in particular in nonliear optaics and in optical fibers.
\end{abstract}

\section{Keywords}

Nonlinear Schrödinger Equation, Bogning-Djeumen Tchaho-Kofané Method, Hybrid Dispersive Optical Solitons, Cubic-Quintic-Septic Law, Strong Dispersions, Nonlinear Optics 


\section{Introduction}

Human beings, in search of well-being, face daily the multiple obstacles (or difficulties) imposed on them by the universe in its complexity. Therefore, a momentum of enormous progress in the advancement of essential knowledge and understanding of the natural phenomena born of these obstacles becomes essential. It is undoubtedly in this context that, for decades, several fields of research have emerged with the aim of providing adequate responses in order to preserve lives and improve living conditions. At the heart of these fields of science, in particular in physics, we can cite among others fluid mechanics, solid state physics, nonlinear optics, plasma physics, data transmission and so on. Since the world around us is intrinsically nonlinear, nonlinear partial differential equations (NLPDEs) appear to be the best adapted and are widely used to describe complex phenomena [1] [2] [3] [4] [5] in these different fields. Among these NLPDEs, those describing the dynamics of dispersive optical solitary wave attract our attention, in particular, the Schrödinger-Hirota equation and the Fokas-Lenells equation. Although these two models have been studied in depth [6]-[11] in the past, several behaviors developed by physical systems whose dynamics are described by these latter (models) still remain to be highlighted in the concern of possible improvement of the living conditions of citizens of the world, for example, the long range worldwide traffic of data (voice, images, etc.) and at very high speed through optical fibers. As part of the most exciting advances in nonlinear science and theoretical physics, one has attracted considerable the attention from many researchers around the globe, including the development of methods [12]-[28] to search for exact solutions of the nonlinear partial differential equations. But very few of these methods offer approximate solutions, or even forced solutions, because these exact solutions are not always easy to construct given that, such requires a deep understanding of mathematics. In this setting, one quickly realizes by visiting the literature that, in the field of nonlinear optics, the theory of optical solitons occupies a spot of choice and, in recent decades, a large amount of results have been highlighted. This mobilization of researchers to produce more results on this subject continues until now. However, in this dynamic, an attention must be more worn paid to the area of dispersive optical solitons because of their significance in the transmission of transoceanic data by optical fibers. These dispersive optical solitons inevitably appearing in systems whose dynamics are governed by nonlinear Schrödinger-Hirota and Fokas-Lenells type equations. An explicit analytical study of these solitons becomes a necessity in the concern of a possible detection of new behaviors which could be developed by propagation media of these dispersive optical solitons. It is on this track that this manuscript sign up, in order to enrich the literature with not only dispersive optical solitons, but also with novel prototypes of hybrid dispersive optical solitons.

Thus, this manuscript aims to constructing and revealing new hybrid dispersives optical soliton solutions of the nonlinear Schrödinger equation presenting the cubic-quintic-septic law coupled with strong dispersions, with a view to 
producing new knowledge regardless of application perspectives. To achieve this, this work is organized as follows: section 2 is devoted to a brief presentation of the model studied. Section 3 focuses its content on the presentation of the main lines of the Bogning-Djeumen Tchaho-Kofané method (BDKm) using the new implicit Bogning' functions (iB-functions) while section 4 is dedicated to the results. The topic on the menu in section 5 is made up of discussions, while, section 6 is dedicated to the conclusion coupled with future prospects

\section{Brief Presentation of the Mathematical Model}

In the context of this work, our choice is carried on the nonlinear Schrödinger equation (NLSE) presenting the cubic-quintic-septic (CQS) law coupled with terms of dispersion of several orders, and which appears in the form [16] [17] [18] [19] [20]

$$
\begin{aligned}
& i q_{t}+i a_{1} q_{x}+a_{2} q_{x x}+i a_{3} q_{x x x}+a_{4} q_{x x x x}+i a_{5} q_{x x x x x}+a_{6} q_{x x x x x x} \\
& +\left(b_{1}|q|^{2}+b_{2}|q|^{4}+b_{3}|q|^{6}\right) q=0 .
\end{aligned}
$$

It is needful to note here that Equation (1) is under its dimensionless form and where $q(x, t)$ holds lieu of the wave profile with complex values while $q_{t}$ represents linear temporal evolution of this profile. The different terms from second to seventh represent intermodal dispersion (IMD), group speed dispersion (GVD), third order dispersion (3OD), fourth order dispersion (4OD), fifth order dispersion (5OD) and the sixth order dispersion (6OD), respectively. As for the eighth, ninth and tenth terms, they together constitute the CQS law. All the coefficients $a_{s},(s=1 ; 2 ; \cdots ; 6)$ and $b_{j},(j=1 ; 2 ; 3)$ are constant real numbers and $i$, an imaginary such that $i^{2}=-1$. From the point of view of theoretical physics and mathematical, Equation (1) remains one of the most relevant and it governs the dynamics of propagation of dispersive optical solitons in nonlinear optical fibers. To be simpler, the NLSE is a sort of reading table for an appropriation of the transmission of light waves through various non-linear supports. This importance granted by theoretical physics and mathematical to this equation sure finds its explanation for the fact that currently, all optical communications used for transcontinental and transoceanic data transfer, are done through long distance optical fibers. In a context of highly dispersive solitons, by the time the group velocity dispersion (GVD) is low, an appearance of the terms IMD, 3OD, 4OD, 5OD and 6OD becomes essential to establish the balance between the non-linearity and dispersion, and thus ensuring the stability of the soliton. It follows for this purpose an improvement in performance during the propagation of solitons over long distances [10] [29]. In the following paragraph, we present the implementation of the BDKm which we will use in section 4 to construct hybrid dispersive optical soliton solutions of Equation (1).

\section{The BDKm Theory Extended to the iB-Functions}

Here, a brief presentation of the iB-functions will be followed by the implemen- 
tation of the BDKm in order to allow the reader to better arm themselves with the rudiments necessary for the proper understanding of the results which will be unearthed in section 4 .

\section{1. iB-Functions}

These iB-functions [25] [30] [31] have been highlighted thanks to the multiple research works [21] [22] [23] [24] [25] [31]-[43] produced for more than a decade. It is during the repeated constructions of solitary wave solutions of certain types of equations in wave mechanics presenting dispersion terms coupled with nonlinear terms (which can be of different orders) via the BDKm, that the fascinating properties of these functions were detected [25] [30] [31]. Under its hyperbolic form, the iB-function is written

$$
J_{n, m}\left(\sum_{i=0}^{p} \alpha_{i} x_{i}\right)=\frac{\sinh ^{m}\left(\sum_{i=0}^{p} \alpha_{i} x_{i}\right)}{\cosh ^{n}\left(\sum_{i=0}^{p} \alpha_{i} x_{i}\right)}=\sinh ^{m}\left(\sum_{i=0}^{p} \alpha_{i} x_{i}\right) \operatorname{sech}^{n}\left(\sum_{i=0}^{p} \alpha_{i} x_{i}\right) .
$$

The member on the left is the implicit form and the member on the right is the explicit form of the function, where $\alpha_{i},(i=0 ; 1 ; 2 ; \cdots ; p)$ are the parameters associated to the independent variables $x_{i},(i=0 ; 1 ; 2 ; \cdots ; p), m$ is the power of the numerator, $n$ that of the denominator. In one dimensional, according to the choice of the parameter $\alpha_{i}$, Equation (2), is reduced under the form

$$
J_{n, m}(\alpha x)=\frac{\sinh ^{m}(\alpha x)}{\cosh ^{n}(\alpha x)}=\sinh ^{m}(\alpha x) \operatorname{sech}^{n}(\alpha x),
$$

where $\alpha$ is a constant associated to the independant variable $x$. We associate here two of the fundamental properties of this function which will be useful in the rest of this manuscript in the respective forms

$$
\frac{\mathrm{d}^{p} J_{n, m}}{\mathrm{~d} x^{p}}=m \alpha \frac{\mathrm{d}^{p-1} J_{n-1, m-1}}{\mathrm{~d} x^{p-1}}-n \alpha \frac{\mathrm{d}^{p-1} J_{n+1, m+1}}{\mathrm{~d} x^{p-1}}
$$

and

$$
J_{n, m}^{p}=J_{n p, m p} .
$$

It is important to note here that this function in its trigonometric form is written as

$$
J_{n, m}(i x)=i^{m} \frac{\sin ^{m}(x)}{\cos ^{n}(x)} .
$$

For a better understanding of the properties of these functions, it is needful to refer to [25] [30] [31] where they are widely explained.

\subsection{Implementation of the BDKm}

The BDKm finds its implementation field in nonlinear physics, wave mechanics, mathematics physics, and others. It is better suited for solving certain types of NPDEs of the form [21] [22] [23] [24] [25] [31]-[43] 


$$
T\left(\phi, \phi_{t}, \phi_{x}, \phi_{t t}, \phi_{x x}, \phi_{t t t}, \phi_{x x x}, \cdots,|\phi|^{2},\left(\phi|\phi|^{2}\right)_{t}, \cdots\right)=0,
$$

where $\phi(x, t)$ is an unknown function to be determined, $T$ is some function of $\phi$ and its derivatives with respect to $x$ and $t$, and $T$ includes the highest order derivatives and the nonlinear terms. Generally, the solution sought is of the form $\lambda_{i j} J_{i, j}\left(\sum_{k=0}^{p} \alpha_{k} x_{k}\right)$. As in the logic of a only variable, we can set the change of variable $\xi=\sum_{k=0}^{p} \alpha_{k} x_{k}$. But in the case where we have a function of $x$ and $t$; $\phi(x, t)$, we can pose the change of variable $\xi=x-v t$. Thus, $\phi(x, t)$ becomes $\phi(\xi)$ where $v$ is the speed of the wave and Equation (7) becomes in these conditions

$$
T_{O D E}\left(\Theta, \Theta^{\prime}, \Theta^{\prime \prime}, \cdots, \Theta^{\prime}|\Theta|^{2}, \cdots\right)=0 .
$$

Equation (8) is an ordinary differential equation(ODE), where $\Theta^{\prime}, \Theta^{\prime \prime}$ represent respectively the first and second derivatives of the envelope $\Theta$ with respect to $\xi$. According to Equation (3), the solution we are trying to construct can be expressed as

$$
\Theta(\xi)=\sum_{i j} \lambda_{i j} J_{j, i}(\eta \xi)
$$

where $\eta$ is a real constant and $\lambda_{i j}$ are the unknown constants to be determined. So, the combination of Equations (9) and (8) gives the main equation

$$
\begin{aligned}
& \sum_{i j n} P_{n}\left(\lambda_{i j}, \eta, v\right) J_{n, 0}(\eta \xi)+\sum_{i j m} Q_{m}\left(\lambda_{i j}, \eta, v\right) J_{m, 1}(\eta \xi)+\sum_{i j k} R_{k}\left(\lambda_{i j}, \eta, v\right) J_{-k, 0}(\eta \xi) \\
& +\sum_{i j l} S_{l}\left(\lambda_{i j}, \eta, v\right) J_{-l, 1}(\eta \xi)+\sum_{i j} Y\left(\lambda_{i j}, \eta, v\right) J_{0,0}(\eta \xi)=0
\end{aligned}
$$

where $i, j, k, l$ are positive natural integers and $n, m$ the real numbers [29] [34] [35]. It can be noted here that Equation (10) is the one from which all the possible analyzes result. The identification of coefficients

$P_{n}\left(\lambda_{i j}, \eta, v\right), Q_{m}\left(\lambda_{i j}, \eta, v\right), R_{k}\left(\lambda_{i j}, \eta, v\right), S_{l}\left(\lambda_{i j}, \eta, v\right), Y\left(\lambda_{i j}, \eta, v\right)$ at zero makes it possible to obtain the ranges of equations whose resolutions could allow to obtain the expressions of the unknown coefficients $\lambda_{i j}$. Solving these series of equations can lead to exact, approximated or forced solutions [34] [35] [36] [37] depending on the models and the form of the considered ansatz. In the case of approximate or forced solutions, the priority in the order of resolution is given to those from the highest clues of $J_{n, 0}(\eta \xi)$, then to those of the highest clues of $J_{m, 1}(\eta \xi)$. But, otherwise we go to those from the coefficients of lowest clues of $J_{-k, 0}(\eta \xi)$ and $J_{-l, 1}(\eta \xi)$. Here, the priority makes reference to the serie that permits to obtain good results or merely that tends more to the sought exact solution. Very often, the series of equations obtained by identify at zero the coefficient of $J_{n, 0}(\eta \xi)$ gives satisfaction. At the opposite, the last serie of equations given by the coefficient of $J_{0,0}(\eta \xi)$ is not very important because it is considered rather like a confused domain for the obtainable good solutions. So, this resolution permits to obtain the possible expressions of the coefficients $\lambda_{i j}$ of Equation (9) as a function of the parameters $\eta, v$, and those supplied by Equa- 
tion (7), and then of the constraints which may result therefrom. Thus, the ansatz given in Equation (9) can be supported by Equation (7) as a solution. Recently, this method was used in [34] [35] and in [41] [42] [43] to construct hybrid solitary waves for the generalized Kuramoto-Sivashinsky, Multi-form solitary wave solutions of the KdV-Burgers-Kuaramoto equation and solitary wave solutions which propagate through transmission media such as electrical lines, respectively. Now, we apply it again and in the following lines, to the NLSE which presents the CQS law coupled with terms of dispersion of several orders.

\section{Results}

We are exploited the BDKm to approach and unearth the new dispersive optical solitons that we qualify as hybrids due to the design and constitution of the ansatz (see Equation (13)) that we will use in this part of the work.

\subsection{Obtention of the Range Equations}

This obtention comes from a rigorous application of the BDKm to Equation (1) preceded by the judicious choice of the mathematical form of the ansatz. Thus, considering the soliton profile in the form

$$
q(x, t)=\psi(\xi) \mathrm{e}^{i \omega t},
$$

where $\xi=x-v t ; \omega$ is the wave number, $v$ the wave speed, we obtain from Equation (1), the equation satisfied by $\psi$ as

$$
\begin{aligned}
& i\left(a_{1}-v\right) \psi_{\xi}+i a_{1}+a_{2} \psi_{\xi \xi}+i a_{3} \psi_{\xi \xi \xi}+a_{4} \psi_{\xi \xi \xi \xi}+i a_{5} \psi_{\xi \xi \xi \xi \xi}+a_{6} \psi_{\xi \xi \xi \xi \xi \xi} \\
& +\left[b_{1} \psi \psi^{*}+b_{2}\left(\psi \psi^{*}\right)^{2}+b_{3}\left(\psi \psi^{*}\right)^{3}-\omega\right] \psi=0,
\end{aligned}
$$

where $\psi^{*}$, denote the complex conjugate of $\psi$. Thus, taking into account the requirements related to the implementation of the $\mathrm{BDKm}$ presented to the previous section and in relation to the $\mathrm{BB}$-function, it is easy to conceive the solution of Equation (12) which we seek to construct as being

$$
\begin{aligned}
\psi(\xi) & =\sum_{s=0}^{1}\left[\beta_{2 s+1} J_{2 s+1,0}(\alpha \xi)+i \gamma_{2 s+1} J_{2 s+1,2 s+1 i}(\alpha \xi)\right] \\
& =a J_{1,0}(\alpha \xi)+i b J_{1,1}(\alpha \xi)+c J_{3,0}(\alpha \xi)+i d J_{3,3}(\alpha \xi),
\end{aligned}
$$

where $a=\beta_{1}, b=\gamma_{1}, c=\beta_{3}$ and $d=\gamma_{3}$ are real constants to be determined, $\alpha$, the inverse of the width of each component of the ansatz and $i$, an imaginary such that $i^{2}=-1$. Let us point out here that, the architecture of Equation (13) reveals a prototype of hybrid dispersive optical solitons. This is because, Equation (13) is a sort of multi-form solitary waves generated by the pooling together of four terms from the two major families of solitary waves, namely, bright and dark type, respectively. To be a little clearer, the first and third terms are both bright type from the large bright family, while, the second and fourth terms represent kink and double-kink respectively, from the large dark family. In summary, Equation (13) can be perceived as being a complex package [25] [30] 
[31] of dispersive optical solitons that can be grouped into two sub-packages: the first sub-package is the real part consisting of two bright solitons which differ in their powers 1 and 3, respectively, and the second sub-package is the imaginary part formed by a kink and double-kink [34] of the big dark family. That said, we continue our analysis by inserting Equation (13) into Equation (12) to obtain the contracted form of the main equation below

$$
\begin{aligned}
& \sum_{s} P_{s}\left(a, b, c, d, a_{k}, b_{r}, \alpha, \omega, v\right) J_{s, 0}(\alpha \xi) \\
& +i \sum_{j} Q_{j}\left(a, b, c, d, a_{k}, b_{r}, \alpha, \omega, v\right) J_{j, 1}(\alpha \xi)=0,
\end{aligned}
$$

where $s \in\{1 ; 2 ; 3 ; 4 ; 5 ; 6 ; 7 ; 8 ; 9 ; 11 ; 13 ; \cdots ; 21\}$;

$j \in\{1 ; 2 ; 3 ; 4 ; 5 ; 6 ; 7 ; 8 ; 9 ; 11 ; 13 ; \cdots ; 21\} ; k \in\{1 ; 2 ; 3 ; 4 ; 5 ; 6\}$ and $r \in\{1 ; 2 ; 3\}$. So, Equation (14) has delivered in its formulation, two ranges of equations in the terms of $J_{s, 0}(\alpha \xi)$, and $J_{j, 1}(\alpha \xi)$, thus constituting the most important ranges (while noting that the imaginary part consists only of the equations from the second range $J_{j, 1}(\alpha \xi)$ ) according to the BDKm theory. Thus, We should limit ourselves to solving only the equations from terms in $J_{s, 0}(\alpha \xi)$. The identification at zero, of the coefficients of the terms in $J_{s, 0}(\alpha \xi)$, $s \in\{1 ; 2 ; 3 ; 4 ; 5 ; 6 ; 7 ; 8 ; 9 ; 11 ; 13 ; \cdots ; 21\}$, gives rise to the serie of range algebraic equations, with unknowns $a, b, c$ and $d$ as follows

the term in $J_{21,0}(\alpha \xi)$,

$$
\left(c^{2}-d^{2}\right)^{3} b_{3} c=0
$$

the term in $J_{19,0}(\alpha \xi)$,

$$
\begin{aligned}
& {\left[6 a c^{6}-12 d^{2} a c^{4}+6 d^{4} a c^{2}+\left(9 d^{2}+6 b d\right) c^{5}\right.} \\
& \left.-\left(18 d^{4}+12 b d^{3}\right) c^{3}+\left(9 d^{6}+6 b d^{5}\right) c\right] b_{3}=0,
\end{aligned}
$$

the term in $J_{17,0}(\alpha \xi)$,

$$
\begin{aligned}
& {\left[21 a^{2} c^{5}-30 a^{2} d^{2} c^{3}+9 d^{4} a^{2} c+\left(45 d^{2}+30 b d\right) a c^{4}-\left(54 d^{4}+36 b d^{3}\right) a c^{2}\right.} \\
& +\left(6 b d^{5}+9 d^{6}\right) a-\left(3 b^{2}+12 b d+9 d^{2}\right) c^{5}+\left(27 d^{4}+60 b d^{3}+18 b^{2} d^{2}\right) c^{3} \\
& \left.-\left(18 d^{6}+48 b d^{5}+15 b^{2} d^{4}\right) c\right] b_{3}=0
\end{aligned}
$$

the term in $J_{15,0}(\alpha \xi)$,

$$
\begin{aligned}
& {\left[\left(35 c^{4}+3 d^{4}-30 d^{2} c^{2}\right) a^{3}+\left(90 d^{2}+60 b d\right) a^{2} c^{3}-\left(54 d^{4}+36 b d^{3}\right) a^{2} c\right.} \\
& -\left(15 b^{2}+60 b d+45 d^{2}\right) a c^{4}+\left(78 d^{4}+120 b d^{3}+36 b^{2} d^{2}\right) a c^{2} \\
& -\left(18 d^{6}+48 b d^{5}+15 b^{2} d^{4}\right) a+\left(3 b^{2}+6 b d+3 d^{2}\right) c^{5} \\
& +\left(60 d^{4}+12 b^{3} d+120 b d^{3}+72 b^{2} d^{2}\right) c^{3} \\
& \left.+\left(66 d^{6}+156 b d^{5}+105 b^{2} d^{4}+20 b^{3} d^{3}\right) c\right] b_{3}+\left(c^{2}-d^{2}\right)^{2} c b_{2}=0
\end{aligned}
$$

the term in $J_{13,0}(\alpha \xi)$, 


$$
\begin{aligned}
& {\left[\left(35 c^{3}-15 d^{2} c\right) a^{4}+\left(90 d^{2}+60 b d\right) a^{3} c^{2}-\left(18 d^{4}+12 b d^{3}\right) a^{3}\right.} \\
& -\left(30 b^{2}+120 b d+84 d^{2}\right) a^{2} c^{3}+\left(123 d^{4}+180 b d^{3}+54 b^{2} d^{2}\right) a^{2} c \\
& +\left(15 b^{2}+30 b d+15 d^{2}\right) a c^{4}-\left(180 d^{4}+36 b^{3} d+360 b d^{3}+216 b^{2} d^{2}\right) a c^{2} \\
& +\left(66 d^{6}+156 b d^{5}+105 b^{2} d^{4}+20 b^{3} d^{3}\right) a+\left(3 b^{4}+45 d^{4}+36 b^{3} d+120 b d^{3}\right. \\
& \left.\left.+108 b^{2} d^{2}\right) c^{3}-\left(126 d^{6}+336 b d^{5}+15 b^{4} d^{2}+315 b^{2} d^{4}+120 b^{3} d^{3}\right) c\right] b_{3} \\
& +\left[5 a c^{4}-6 d^{2} a c^{2}+d^{4} a+\left(6 d^{2}+4 b d\right) c^{3}-\left(6 d^{4}+4 b d^{3}\right) c\right] b_{2}=0,
\end{aligned}
$$

the term in $J_{11,0}(\alpha \xi)$,

$$
\begin{aligned}
& {\left[\left(21 c^{2}-3 d^{2}\right) a^{5}+\left(37 d^{2}+25 b d\right) a^{4} c+\left(45 d^{4}+60 b d^{3}+18 b^{2} d^{2}\right) a^{3}\right.} \\
& -\left(30 b^{2}+120 b d+84 d^{2}\right) a^{3} c^{2}+\left(30 b^{2}+60 b d+30 d^{2}\right) a^{2} c^{3} \\
& -\left(180 d^{4}+36 b^{3} d+360 b d^{3}+216 b^{2} d^{2}\right) a^{2} c \\
& +\left(9 b^{4}+135 d^{4}+108 b^{3} d+360 b d^{3}+324 b^{2} d^{2}\right) a c^{2} \\
& -\left(126 d^{6}+336 b d^{5}+15 b^{4} d^{2}+315 b^{2} d^{4}+120 b^{3} d^{3}\right) a \\
& -\left(6 b^{4}+18 d^{4}+36 b^{3} d+60 b d^{3}+72 b^{2} d^{2}\right) c^{3} \\
& \left.+\left(126 d^{6}+6 b^{5} d+240 b d^{5}+75 b^{4} d^{2}+525 b^{2} d^{4}+300 b^{3} d^{3}\right) c\right] b_{3} \\
& +\left[10 a^{2} c^{3}-6 d^{2} a^{2} c+\left(18 d^{2}+12 b d\right) a c^{2}-\left(6 d^{4}+4 b d^{3}\right) a\right. \\
& \left.-\left(2 b^{2}+8 b d+6 d^{2}\right) c^{3}+\left(15 d^{4}+20 b d^{3}+6 b^{2} d^{2}\right) c\right] b_{2}=0,
\end{aligned}
$$

the term in $J_{9,0}(\alpha \xi)$,

$$
\begin{aligned}
& \quad\left[7 a^{6} c+\left(d^{2}+b d\right) a^{5}-\left(15 b^{2}+48 b d+57 d^{2}\right) a^{4} c\right. \\
& -\left(60 d^{4}+12 b^{3} d+120 b d^{3}+72 b^{2} d^{2}\right) a^{3}+\left(30 b^{2}+60 b d+30 d^{2}\right) a^{3} c^{2} \\
& +\left(9 b^{4}+135 d^{4}+108 b^{3} d+360 b d^{3}+324 b^{2} d^{2}\right) a^{2} c \\
& -\left(18 b^{4}+54 d^{4}+108 b^{3} d+180 b d^{3}+216 b^{2} d^{2}\right) a c^{2} \\
& +\left(126 d^{6}+6 b^{5} d+420 b d^{5}+75 b^{4} d^{2}+525 b^{2} d^{4}+300 b^{3} d^{3}\right) a \\
& +\left(3 b^{4}+3 d^{4}+12 b^{3} d+12 b d^{3}+18 b^{2} d^{2}\right) c^{3} \\
& \left.\quad-\left(b^{6}+84 d^{6}+24 b^{5} d+156 b d^{5}+150 b^{4} d^{2}+525 b^{2} d^{4}+400 b^{3} d^{3}\right) c\right] b_{3} \\
& +\left[-2 d^{2} a^{3}+10 a^{3} c^{2}+\left(18 d^{2}+12 b d\right) a^{2} c-\left(6 b^{2}+24 b d+18 d^{2}\right) a c^{2}\right. \\
& +\left(15 d^{4}+20 b d^{3}+6 b^{2} d^{2}\right) a+\left(2 b^{2}+4 b d+2 d^{2}\right) c^{3} \\
& \left.\quad-\left(20 d^{4}+4 b^{3} d+40 b d^{3}+24 b^{2} d^{2}\right) c\right] b_{2}+\left(c^{2}-d^{2}\right) c b_{1}=0, \\
& \text { the term in } J_{8,0}(\alpha \xi),
\end{aligned}
$$




$$
2520 \alpha^{5} a_{5} d=0,
$$

the term in $J_{7,0}(\alpha \xi)$,

$$
\begin{aligned}
& {\left[a^{7}-\left(3 b^{2}+12 b d+9 d^{2}\right) a^{5}+\left(15 b^{2}+30 b d+15 d^{2}\right) a^{4} c\right.} \\
& +\left(3 b^{4}+45 d^{4}+36 b^{3} d+120 b d^{3}+108 b^{2} d^{2}\right) a^{3} \\
& -\left(18 b^{4}+54 d^{4}+108 b^{3} d+180 b d^{3}+216 b^{2} d^{2}\right) a^{2} c \\
& +\left(9 b^{4}+9 d^{4}+36 b^{3} d+36 b d^{3}+54 b^{2} d^{2}\right) a c^{2} \\
& -\left(b^{6}+84 d^{6}+24 b^{5} d+156 b d^{5}+150 b^{4} d^{2}+525 b^{2} d^{4}+400 b^{3} d^{3}\right) a \\
& \left.+\left(3 b^{6}+36 d^{6}+36 b^{5} d+102 b d^{5}+141 b^{4} d^{2}+306 b^{2} d^{4}+282 b^{3} d^{3}\right) c\right] b_{3} \\
& +\left[4 a^{4} c+\left(6 d^{2}+4 b d\right) a^{3}-\left(4 b^{2}+16 b d+12 d^{2}\right) a^{2} c+\left(2 b^{2}+4 b d+2 d^{2}\right) a c^{2}\right. \\
& \left.-\left(20 d^{4}+4 b^{3} d+40 b d^{3}+24 b^{2} d^{2}\right) a\right] b_{2}+\left[3 a c^{2}-d^{2} a+\left(3 d^{2}+2 b d\right) c\right] b_{1} \\
& -720 \alpha^{6} a_{6} a+\left(360 \alpha^{4} a_{4}+29880 \alpha^{6} a_{6}\right) c=0,
\end{aligned}
$$

the term in $J_{6,0}(\alpha \xi)$,

$$
-120 \alpha^{5} a_{5} b-\left(60 \alpha^{3} a_{3}+3480 \alpha^{5} a_{5}\right) d=0,
$$

the term in $J_{5,0}(\alpha \xi)$,

$$
\begin{aligned}
& {\left[\left(3 b^{2}+6 b d+3 d^{2}\right) a^{5}-\left(6 b^{4}+18 d^{4}+36 b^{3} d+60 b d^{3}+72 b^{2} d^{2}\right) a^{3}\right.} \\
& +\left(9 b^{4}+9 d^{4}+36 b^{3} d+36 b d^{3}+54 b^{2} d^{2}\right) a^{2} c \\
& +\left(3 b^{6}+36 d^{6}+36 b^{5} d+102 b d^{5}+141 b^{4} d^{2}+306 b^{2} d^{4}+282 b^{3} d^{3}\right) a \\
& \left.-\left(3 b^{6}+9 d^{6}+24 b^{5} d+48 b d^{5}+75 b^{4} d^{2}+105 b^{2} d^{4}+120 b^{3} d^{3}\right) c\right] b_{3} \\
& +\left[a^{5}-\left(2 b^{2}+8 b d+6 d^{2}\right) a^{3}+\left(6 b^{2}+12 b d+6 d^{2}\right) a^{2} c\right. \\
& +\left(b^{4}+15 d^{4}+12 b^{3} d+40 b d^{3}+33 b^{2} d^{2}\right) a-\left(2 b^{4}+6 d^{4}+12 b^{3} d\right. \\
& \left.\left.+20 b d^{3}+24 b^{2} d^{2}\right) c\right] b_{2}+\left[3 a^{2} c+\left(3 d^{2}+2 b d\right) a-\left(b^{2}+4 b d+3 d^{2}\right) c\right] b_{1} \\
& +\left(24 \alpha^{4} a_{4}+840 \alpha^{6} a_{6}\right) a-\left(12 \alpha^{2} a_{2}+408 \alpha^{4} a_{4}+11172 \alpha^{6} a_{6}\right) c=0,
\end{aligned}
$$

the term in $J_{4,0}(\alpha \xi)$,

$$
\left(6 \alpha^{3} a_{3}+120 \alpha^{5} a_{5}\right) b+\left[3 \alpha\left(v-a_{1}\right)+66 \alpha^{3} a_{3}+1128 \alpha^{5} a_{5}\right] d=0,
$$

the term in $J_{3,0}(\alpha \xi)$,

$$
\begin{aligned}
& {\left[\left(3 b^{4}+3 d^{4}+12 b^{3} d+12 b d^{3}+18 b^{2} d^{2}\right) a^{3}-\left(3 b^{6}+9 d^{6}\right.\right.} \\
& \left.+24 b^{5} d+48 b d^{5}+75 b^{4} d^{2}+105 b^{2} d^{4}+120 b^{3} d^{3}\right) a \\
& \left.+\left(b^{6}+d^{6}+6 b^{5} d+6 b d^{5}+15 b^{4} d^{2}+15 b^{2} d^{4}+20 b^{3} d^{3}\right) c\right] b_{3} \\
& +\left[\left(2 b^{2}+4 b d+2 d^{2}\right) a^{3}-\left(2 b^{4}+6 d^{4}+12 b^{3} d+20 b d^{3}+24 b^{2} d^{2}\right) a\right.
\end{aligned}
$$




$$
\begin{aligned}
& \left.+\left(b^{4}+d^{4}+4 b^{3} d+4 b d^{3}+6 b^{2} d^{2}\right) c\right] b_{2}+\left[a^{3}-\left(b^{2}+4 b d+3 d^{2}\right) a\right. \\
& \left.+\left(b^{2}+2 b d+d^{2}\right) c\right] b_{1}-\left(2 \alpha^{2} a_{2}+20 \alpha^{4} a_{4}+182 \alpha^{6} a_{6}\right) a \\
& +\left(9 \alpha^{2} a_{2}+81 \alpha^{4} a_{4}+729 \alpha^{6} a_{6}-\omega\right) c=0,
\end{aligned}
$$

the term in $J_{2,0}(\alpha \xi)$,

$$
\left[\alpha\left(v-a_{1}\right)-4 \alpha^{3} a_{3}-16 \alpha^{5} a_{5}\right] b+\left[3 \alpha\left(v-a_{1}\right)-12 \alpha^{3} a_{3}-48 \alpha^{5} a_{5}\right] d=0,
$$

the term in $J_{1,0}(\alpha \xi)$,

$$
\begin{aligned}
& {\left[\left(b^{6}+d^{6}+6 b^{5} d+6 b d^{5}+15 b^{4} d^{2}+15 b^{2} d^{4}+20 b^{3} d^{3}\right) b_{3}\right.} \\
& +\left(b^{4}+d^{4}+4 b^{3} d+4 b d^{3}+6 b^{2} b^{2}\right) b_{2} \\
& \left.+\left(b^{2}+2 b d+d^{2}\right) b_{1}+\alpha^{2} a_{2}+\alpha^{4} a_{4}+\alpha^{6} a_{6}-\omega\right] a=0 .
\end{aligned}
$$

The continuation of this study focuses on the analysis and resolution of the range Equations (15) to (29). However, it is useful to notice that, when one fixes one of the values of the unknowns coefficients, $a, b, c$ or $d$, the major term varies. For instance, if $c=0$ and $d=0$, Equations (15) to (22) are verified and only Equations (23) to (29) are those on which next investigations will also refer to.

\subsection{Analysis of the Range Equations}

At first glance, it is easy to see that Equations (15) and (22), respectively, lead to

$$
c= \pm d, \text { or } b_{3}=0 \text {, or } c=0
$$

and

$$
a_{5}=0 \text {, or } d=0 \text {. }
$$

Thus, the choice of the different families of solutions to be constructed, in the following, will be based around both conditions (30) and (31). It appears that the cases $c= \pm d, b_{3}=0$ and $a_{5}=0$ open the way to many speculations that can guide this choice. Thus, for $b_{3}=0$, Equations (15), (16) and (17) are verified, while, Equation (18) leads to $\left(c^{2}-d^{2}\right)^{2} c b_{2}=0$. What highlights a possible additional condition in the choice of solutions to be constructed: $b_{2}=0$. If in addition, we associate with $b_{3}=0$, the condition $c= \pm d$, then, Equation (19) is verified while Equations (20) and (21) due to their complexities require to choose $b_{2}=0$.

By summarizing all this mainly to $b_{3}=b_{2}=a_{5}=0$, Equations (15) to (20), (22) are verified while Equations (21), (23)-(29) are respectively scaled down to

$$
\begin{gathered}
\left(c^{2}-d^{2}\right) c b_{1}=0 \\
{\left[\left(3 c^{2}-d^{2}\right) b_{1}-720 \alpha^{6} a_{6}\right] a+\left[360 \alpha^{4} a_{4}+29880 \alpha^{6} a_{6}+\left(3 d^{2}+2 b d\right) b_{1}\right] c=0} \\
a_{3}=0
\end{gathered}
$$




$$
\begin{gathered}
3 b_{1} a^{2} c+\left(3 d^{2}+2 b d\right) b_{1} a+\left(24 \alpha^{4} a_{4}+840 \alpha^{6} a_{6}\right) a \\
-\left[12 \alpha^{2} a_{2}+405 \alpha^{4} a_{4}+11172 \alpha^{6} a_{6}+\left(b^{2}+4 b d+3 d^{2}\right) b_{1}\right] c=0 \\
v=a_{1}, \\
b_{1} a^{3}-\left[\left(b^{2}+4 b d+3 d^{2}\right) b_{1}+2 \alpha^{2} a_{2}+20 \alpha^{4} a_{4}+182 \alpha^{6} a_{6}\right] a \\
+\left[9 \alpha^{2} a_{2}+81 \alpha^{4} a_{4}+729 \alpha^{6} a_{6}+\left(b^{2}+2 b d+d^{2}\right) b_{1}-\omega\right] c=0, \\
v=a_{1}, \\
\omega=\left(b^{2}+2 b d+d^{2}\right) b_{1}+\alpha^{2} a_{2}+\alpha^{4} a_{4}+\alpha^{6} a_{6} .
\end{gathered}
$$

It is very important to note here that this analysis gives rise to two families of non-trivial solutions to which we will focus all of our attention throughout the following paragraph.

\subsection{Analytical Hybrid Dispersive Optical Solitons}

In this subsection, we group the obtained solutions into two large families that we name: First family of solutions and Second family of solutions. The first family is incorporated of four subfamilies among which the first subfamily alone account four sub-subfamilies of solution that we have named: first subfamily 1 of solutions, second subfamily 1 of solutions (see paragraph 4.3.1.-1)), first subfamily 2 of solutions and second subfamily 2 of solutions (see paragraph 4.3.1.-2)). As for the second family of solutions, it has three subfamilies of solutions among which the third subfamily alone comprises two sub-subfamilies given by the sub-paragraphs 4.3.2.-3)-a) and 4.3.2.-3)-b), respectively.

\subsubsection{First Family of Solutions; Case: $b_{3}=b_{2}=a_{5}=a_{3}=0, \quad v=a_{1}$}

In this case, the substitution of Equation (39) in Equation (37) gives

$$
\begin{aligned}
& b_{1} a^{3}-\left[\left(b^{2}+4 b d+3 d^{2}\right) b_{1}+2 \alpha^{2} a_{2}+20 \alpha^{4} a_{4}+182 \alpha^{6} a_{6}\right] a \\
& +\left(8 \alpha^{2} a_{2}+80 \alpha^{4} a_{4}+728 \alpha^{6} a_{6}\right) c=0
\end{aligned}
$$

Equation (33) and Equation (40) give, respectively,

$\left(3 d^{2}+2 b d\right) b_{1}=\frac{\left[\left(d^{2}-3 c^{2}\right) b_{1}+720 \alpha^{6} a_{6}\right] a-\left(360 \alpha^{4} a_{4}+29880 \alpha^{6} a_{6}\right) c}{c} ; c \neq 0$

and

$$
\begin{aligned}
& \left(b^{2}+4 b d+3 d^{2}\right) b_{1} \\
& =\frac{b_{1} a^{3}-\left(2 \alpha^{2} a_{2}+20 \alpha^{4} a_{4}+182 \alpha^{6} a_{6}\right) a+\left(8 \alpha^{2} a_{2}+80 \alpha^{4} a_{4}+720 \alpha^{6} a_{6}\right) c}{a} ; a \neq 0 .
\end{aligned}
$$

Taking into account Equations (41) and (42) in Equation (35) leads to an equation with three unknown coefficients $a, c$ and $d$ as follows

$$
\begin{aligned}
& \left(d^{2}-c^{2}\right) b_{1} a^{3}+720 \alpha^{6} a_{6} a^{3}-\left(360 \alpha^{4} a_{4}+29880 \alpha^{6} a_{6}\right) a^{2} c \\
& -\left(10 \alpha^{2} a_{2}+388 \alpha^{4} a_{4}+10990 \alpha^{6} a_{6}\right) a c^{2}-\left(8 \alpha^{2} a_{2}+80 \alpha^{4} a_{4}+728 \alpha^{6} a_{6}\right) c^{3}=0 .
\end{aligned}
$$


One can notices here that, Equation (43) is the main equation on which we will derive the different first sub-families of solutions of the first family.

1) First subfamily of the first family of solutions; case:

$b_{3}=b_{2}=a_{5}=a_{3}=0, c=d \neq 0$

Under these conditions, Equation (43) leads to a third degree equation to two unknown coefficients $a$ and $d$ in the form

$$
\begin{aligned}
& 360 \alpha^{4} a_{6} a^{3}-\left(180 \alpha^{2} a_{4}+14940 \alpha^{4} a_{6}\right) a^{2} d-\left(5 a_{2}+194 \alpha^{2} a_{4}+5495 \alpha^{4} a_{6}\right) a d^{2} \\
& -\left(4 a_{2}+40 \alpha^{2} a_{4}+364 \alpha^{4} a_{6}\right) d^{3}=0
\end{aligned}
$$

Facing a third degree equation to two unknown coefficients $a$ and $d$, and unable to identify an obvious solution of Equation (44), one must, to solve this, fixes one of the unknowns $a$ or $d$ by associating two additional conditions ( $a_{6}=0$ or $a_{2}=-10 \alpha^{2} a_{4}-91 \alpha^{4} a_{6}$ ) obtained by equating at zero the coefficients of $a^{3}$ and $d^{b}$, respectively.

- First associated conditions 1: $c=d=\lambda_{1}, \lambda_{1} \in \mathfrak{R}^{*}$ and $a_{6}=0$

Under these conditions, Equation (44) leads to a quadratic equation in unknown $a$ as follows

$$
m_{1} a^{2}+n_{1} a+p_{1}=0
$$

with $m_{1}=180 \alpha^{2} a_{4}, n_{1}=\left(5 a_{2}+194 \alpha^{2} a_{4}\right) \lambda_{1}, p_{1}=\left(4 a_{2}+40 \alpha^{2} a_{4}\right) \lambda_{1}^{2}$. Solving in the case of the positive discriminant allows Equation (45) to support the coefficient a below as solution

$$
a=\frac{-\left(5 a_{2}+194 \alpha^{2} a_{4}\right) \lambda_{1} \pm\left|\lambda_{1}\right| \sqrt{25 a_{2}^{2}-940 \alpha^{2} a_{2} a_{4}+8836 \alpha^{4} a_{4}^{2}}}{360 \alpha^{2} a_{4}} .
$$

From Equation (46), one can notice that the amplitude of the first term of the ansatz (13) is a function of the coefficients of the dispersion terms of order three, order four and the width at half height of the soliton only. Thus, we can easily obtain from Equation (41), the coefficient $b$ in the form

$$
b=-\frac{3}{2} \lambda_{1}-a-180 \alpha^{4} \frac{a_{4}}{\lambda_{1} b_{1}}
$$

and the first subfamily 1 of the first family of solutions comes in the form

$$
\begin{aligned}
q(x, t)= & {\left[\frac{-\left(5 a_{2}+194 \alpha^{2} a_{4}\right) \lambda_{1} \pm\left|\lambda_{1}\right| \sqrt{25 a_{2}^{2}-940 \alpha^{2} a_{2} a_{4}+8836 \alpha^{4} a_{4}^{2}}}{360 \alpha^{2} a_{4}} J_{1,0}(\alpha \xi)\right.} \\
& \left.+i\left(-\frac{3}{2} \lambda_{1}-a-180 \alpha^{4} \frac{a_{4}}{\lambda_{1} b_{1}}\right) J_{1,1}(\alpha \xi)+\lambda_{1} J_{3,0}(\alpha \xi)+i \lambda_{1} J_{3,3}(\alpha \xi)\right] \mathrm{e}^{i \omega t},
\end{aligned}
$$

where $b_{1} \neq 0 ; a_{4} \neq 0$ and $a$ is given by Equation (46).

- Second associated conditions 1: $c=d, a=\gamma_{1}, \gamma_{1} \in \mathfrak{R}^{*}$ and

$$
a_{2}=-10 \alpha^{2} a_{4}-91 \alpha^{4} a_{6}
$$

In this context, Equation (44) becomes a quadratic equation in unknown $d$ below 


$$
m_{2} d^{2}+n_{2} d+p_{2}=0
$$

with $m_{2}=4 a_{4}+140 \alpha^{2} a_{6}, \quad n_{2}=\left(5 a_{4}+415 \alpha^{2} a_{6}\right) \gamma_{1}, \quad p_{2}=-10 \alpha^{2} a_{6} \gamma_{1}^{2}$ and its resolution reveals the expression of the unknown $d$ in the form

$$
\begin{aligned}
& d=\frac{-\left(5 a_{4}+415 \alpha^{2} a_{6}\right) \gamma_{1} \pm\left|\gamma_{1}\right| \sqrt{25 a_{4}^{2}+4310 \alpha^{2} a_{4} a_{6}+177825 \alpha^{4} a_{6}^{2}}}{8 a_{4}+280 \alpha^{2} a_{6}} ; \\
& a_{4} \neq-35 \alpha^{2} a_{6} .
\end{aligned}
$$

From Equation (50), one can notice that the amplitude of the second and fourth terms of the ansatz (13) is a function of the coefficients of the dispersion terms of order four, order six and the width at half height of the soliton only. So, from Equation (41), we obtain the coefficient $b$ as

$$
b=-\frac{3}{2} d-\frac{\left(d^{2} b_{1}-360 \alpha^{6} a_{6}\right) \gamma_{1}+\left(180 \alpha^{4} a_{4}+14940 \alpha^{6} a_{6}\right) d}{d^{2} b_{1}} ; b_{1} \neq 0,
$$

and the second subfamily 1 of the first family of solutions is

$$
\begin{aligned}
q(x, t)= & {\left[\gamma_{1} J_{1,0}(\alpha \xi)-i\left(\frac{3}{2} d+\frac{\left(d^{2} b_{1}-360 \alpha^{6} a_{6}\right) \gamma_{1}+\left(180 \alpha^{4} a_{4}+14940 \alpha^{6} a_{6}\right) d}{d^{2} b_{1}}\right) J_{1,1}(\alpha \xi)\right.} \\
& \left.+d J_{3,0}(\alpha \xi)+i d J_{3,3}(\alpha \xi)\right] \mathrm{e}^{i \omega t},
\end{aligned}
$$

where $d$ is given by Equation (50). One should note here that, Equations (48) and (52) are the first prototypes of hybrid dispersive optical solitons which we believe the last two terms resulting from Equation (13) reveal a competition of equal amplitudes among them. This competition is disclosed by the sequence $\left(c^{2}-d^{2}\right)^{k} b_{k}, k \in\{1 ; 2 ; 3\}$ which can be identified across Equations (21), (18) and (15), and therefore their structure has suggested the choice of $c=d$ condition.

2) Second subfamily of the first family of solutions; case:

$b_{3}=b_{2}=a_{5}=a_{3}=0, c=-d \neq 0$

From these conditions, Equation (43) becomes

$$
\begin{aligned}
& 360 \alpha^{4} a_{6} a^{3}+\left(180 \alpha^{2} a_{4}+14940 \alpha^{4} a_{6}\right) a^{2} d-\left(5 a_{2}+194 \alpha^{2} a_{4}+5495 \alpha^{4} a_{6}\right) a d^{2} \\
& +\left(4 a_{2}+40 \alpha^{2} a_{4}+364 \alpha^{4} a_{6}\right) d^{3}=0 .
\end{aligned}
$$

We note here that we face the same difficulties as in the case of Equation (44). Which suppose that we will use the same approach for its resolution.

- First associated conditions 2: $d=-c=\lambda_{2}, \lambda_{2} \in \mathfrak{R}^{*}$ and $a_{6}=0$

Taking these conditions into account in Equation (53) gives rise to a quadratic equation in $a$ in the form

$$
m_{3} a^{2}+n_{3} a+p_{3}=0
$$

with $m_{3}=180 \alpha^{2} a_{4}, \quad n_{3}=-\left(5 a_{2}+194 \alpha^{2} a_{4}\right) \lambda_{2}, \quad p_{3}=\left(4 a_{2}+40 \alpha^{2} a_{4}\right) \lambda_{2}^{2}$. Solving Equation (54) unveils the sought unknown $a$ as being 


$$
a=\frac{\left(5 a_{2}+194 \alpha^{2} a_{4}\right) \lambda_{2} \pm\left|\lambda_{2}\right| \sqrt{25 a_{2}^{2}-940 \alpha^{2} a_{2} a_{4}+8836 \alpha^{4} a_{4}^{2}}}{360 \alpha^{2} a_{4}} ; a_{4} \neq 0
$$

and thereafter, Equation (41) gives

$$
b=-\frac{3}{2} \lambda_{2}+a-180 \alpha^{4} \frac{a_{4}}{\lambda_{2} b_{1}} ; b_{1} \neq 0,
$$

and the first subfamily 2 of the first family of solutions is given by

$$
\begin{aligned}
q(x, t) & =\left[\frac{\left(5 a_{2}+194 \alpha^{2} a_{4}\right) \lambda_{2} \pm\left|\lambda_{2}\right| \sqrt{25 a_{2}^{2}-940 \alpha^{2} a_{2} a_{4}+8836 \alpha^{4} a_{4}^{2}}}{360 \alpha^{2} a_{4}} J_{1,0}(\alpha \xi)\right. \\
& \left.+i\left(-\frac{3}{2} \lambda_{2}+a-180 \alpha^{4} \frac{a_{4}}{\lambda_{2} b_{1}}\right) J_{1,1}(\alpha \xi) i-\lambda_{2} J_{3,0}(\alpha \xi) i+i \lambda_{2} J_{3,3}(\alpha \xi)\right] \mathrm{e}^{i \omega t},
\end{aligned}
$$

where $a$ is given by Equation (55).

- Second associated conditions 2: $c=-d, a=\gamma_{2}, \gamma_{2} \in \mathfrak{R}^{*}$ and $a_{2}=-10 \alpha^{2} a_{4}-91 \alpha^{4} a_{6}$

Under these conditions, Equation (53) takes the quadratic form in unknown $d$

$$
m_{4} d^{2}+n_{4} d+p_{4}=0
$$

with $m_{4}=4 a_{4}+140 \alpha^{2} a_{6}, \quad n_{4}=-\left(5 a_{4}+415 \alpha^{2} a_{6}\right) \gamma_{2}, \quad p_{4}=-10 \alpha^{2} a_{6} \gamma_{2}^{2}$ and its solution is given by

$$
\begin{aligned}
& d=\frac{\left(5 a_{4}+415 \alpha^{2} a_{6}\right) \gamma_{2} \pm\left|\gamma_{2}\right| \sqrt{25 a_{4}^{2}+4310 \alpha^{2} a_{4} a_{6}+177825 \alpha^{4} a_{6}^{2}}}{8 a_{4}+280 \alpha^{2} a_{6}} ; \\
& a_{4} \neq-35 \alpha^{2} a_{6} .
\end{aligned}
$$

From Equation (59), one can notice that the amplitude of the third and of the sixth terms of the ansatz (13) is a function of the coefficients of the dispersion terms of order four, order six and of the width at half height of the soliton only. So, from Equation (41), we obtain the coefficient $b$ as

$$
b=-\frac{3}{2} d+\frac{\left(d^{2} b_{1}-360 \alpha^{6} a_{6}\right) \gamma_{2}+\left(180 \alpha^{4} a_{4}+14940 \alpha^{6} a_{6}\right) d}{d^{2} b_{1}} ; b_{1} \neq 0
$$

and the second subfamily 2 of the first family of solutions is

$$
\begin{aligned}
q(x, t)= & {\left[\gamma_{2} J_{1,0}(\alpha \xi)+i\left(-\frac{3}{2} d+\frac{\left(d^{2} b_{1}-360 \alpha^{6} a_{6}\right) \gamma_{2}+\left(180 \alpha^{4} a_{4}+14940 \alpha^{6} a_{6}\right) d}{d^{2} b_{1}}\right) J_{1,1}(\alpha \xi)\right.} \\
& \left.-d J_{3,0}(\alpha \xi)+i d J_{3,3}(\alpha \xi)\right] \mathrm{e}^{i \omega t},
\end{aligned}
$$

where $d$ is given by Equation (59). One should also note here that, Equations (57) and (61) are the second prototypes of hybrid dispersive optical solitons which we believe the last two terms resulting from equation (13) reveal a competition of opposite amplitudes among them. This competition is disclosed by the sequence 
$\left(c^{2}-d^{2}\right)^{k} b_{k}, k \in\{1 ; 2 ; 3\}$ which can be identified across Equations (21), (18) and (15), and therefore their structure has suggested this time the choice of $c=-d$ condition.

\section{3) Third subfamily of the first family of solutions; case:}

$b_{1} \neq 0 ; b_{3}=b_{2}=a_{5}=a_{3}=0, d \neq c=0$

For the additional condition $d \neq c=0$, Equation (32) is verified, while, solving Equations (33), (35) and (37) successively give

$$
\begin{gathered}
d= \pm \sqrt{-720 \alpha^{6} \frac{a_{6}}{b_{1}}}, \\
b= \pm \frac{55 \alpha^{3} a_{6}-\alpha a_{4}}{60 b_{1} a_{6}} \sqrt{-720 b_{1} a_{6}} ; a_{6} \neq 0, \\
a= \pm \sqrt{b^{2}+4 b d+3 d^{2}+\frac{2 \alpha^{2} a_{2}+20 \alpha^{4} a_{4}+182 \alpha^{6} a_{6}}{b_{1}}},
\end{gathered}
$$

with $a_{6} b_{1} \prec 0, d$ and $b$ in Equation (62) are given by Equation (63) and Equation (64), respectively. So, the third subfamily of the first family of solutions is written as follows

$$
\begin{aligned}
q(x, t)= & {\left[ \pm \sqrt{b^{2}+4 b d+3 d^{2}+\frac{2 \alpha^{2} a_{2}+20 \alpha^{4} a_{4}+182 \alpha^{6} a_{6}}{b_{1}}} J_{1,0}(\alpha \xi)\right.} \\
& \left. \pm i \frac{55 \alpha^{3} a_{6}-\alpha a_{4}}{60 b_{1} a_{6}} \sqrt{-720 b_{1} a_{6}} J_{1,1}(\alpha \xi) \pm i \sqrt{-720 \alpha^{6} \frac{a_{6}}{b_{1}}} J_{3,3}(\alpha \xi)\right] \mathrm{e}^{i \omega t},
\end{aligned}
$$

with $a_{6} b_{1} \prec 0$, the constraint given by Equation (39), $d$ and $b$ are given by Equations (62) and (63), respectively. Equation (65) is a hybrid solution which puts in competition a bright represented by the first term, a kink and a double-kink represented by the second and the third term, respectively. The big dark family (kink and double-kink) outnumber the big bright family (first term). This suggests a tendency to produce a hybrid solution with a strong kink, double-kink or kink-double-kink character as a function of the values of the coefficients $a, b, d, \alpha, \omega, v$ of the wave and the parameters $a_{k}, k \in\{1 ; 2 ; 3 ; 4 ; 5 ; 6\}$ and $b_{r}, r \in\{1 ; 2 ; 3\}$ of system.

\section{4) Fourth subfamily of the first family of solutions; case:}

$b_{3}=b_{2}=a_{5}=a_{3}=0, c \neq d \neq b_{1}=0$

In the context $b_{2}=b_{3}=a_{3}=a_{5}=0$ coupled with the additional condition $c \neq d \neq b_{1}=0$, the Equations (32), (33), (35), (37) and (39) in their different combinations lead to the following constraint

$$
4 a_{2}^{2}+199 \alpha^{2} a_{2} a_{4}+5208 \alpha^{4} a_{2} a_{6}+1590 \alpha^{4} a_{4}^{2}+62909 \alpha^{6} a_{4} a_{6}+44084 \alpha^{8} a_{6}^{2}=0 .
$$

So, the fourth subfamily of the first family of solutions takes the form

$$
q(x, t)=\left[a J_{1,0}(\alpha \xi)+i b J_{1,1}(\alpha \xi)+c J_{3,0}(\alpha \xi)+i d J_{3,3}(\alpha \xi)\right] \mathrm{e}^{i \omega t},
$$

with $\omega=\alpha^{2} a_{2}+\alpha^{4} a_{4}+\alpha^{6} a_{6}, \quad v=a_{1}, \quad a \in \mathfrak{R}^{*}, \quad b \in \mathfrak{R}^{*}, \quad c \in \mathfrak{R}^{*}, \quad d \in \mathfrak{R}^{*}$ and the constraints given by Equation (66). It is important to emphasize here that, 
this fourth subfamily of solutions is that of a part of the linear dispersive equation because all the nonlinear effects as well as the dispersion effects of order three and order five are neglected. Which would suppose that, this mode is marginal with respect to these different effects.

4.3.2. Second Family of Solutions; Case: $c=0, d=0$

For $c=0, d=0$, Equations (15) to (22) are verified, while, Equations (23) to (29) are reduced to, respectively

$$
\begin{gathered}
{\left[\left(a^{2}-b^{2}\right)^{3} b_{3}-720 \alpha^{6} a_{6}\right] a=0,} \\
-120 \alpha^{5} a_{5} b=0, \\
{\left[\left(a^{2}-b^{2}\right)^{2}\left(b_{2}+3 b_{3} b^{2}\right)+840 \alpha^{6} a_{6}+24 \alpha^{4} a_{4}\right] a=0,} \\
\left(6 \alpha^{3} a_{3}+120 \alpha^{5} a_{5}\right) b=0, \\
{\left[\left(a^{2}-b^{2}\right)\left(b_{1}+2 b_{2} b^{2}+3 b_{3} b^{4}\right)-182 \alpha^{6} a_{6}-20 \alpha^{4} a_{4}-2 \alpha^{2} a_{2}\right] a=0,} \\
{\left[\alpha\left(v-a_{1}\right)-4 \alpha^{3} a_{3}-16 \alpha^{5} a_{5}\right] b=0,} \\
{\left[b^{2}\left(b_{1}+b_{2} b^{2}+b_{3} b^{4}\right)+\alpha^{6} a_{6}+\alpha^{4} a_{4}+\alpha^{2} a_{2}-\omega\right] a=0,}
\end{gathered}
$$

From Equation (69), It easily appears that

$$
a_{5}=0 \text { or } b=0 \text {, }
$$

consequently, Equation (71) and Equation (73) lead to, respectively

$$
a_{3}=0 \text { or } b=0 \text {, }
$$

and

$$
v=a_{1} \text { or } b=0 .
$$

To the look of the structure of Equations (68), (70) and (72) which offer the different sequences of the powers of the difference of two squares: $\left(a^{2}-b^{2}\right)^{k} b_{k}$, where $k \in\{1 ; 2 ; 3\}$, the introduction of an additional condition becomes necessary for this second large family of solutions of the Equation (1): $a= \pm b$. Under these considerations, and in order to construct non-trivial solutions, we must consider the following three cases: $a_{5}=a_{3}=0, v=a_{1}, b \neq 0 ; a_{5} \neq 0, a_{3} \neq 0$, $v \neq a_{1}, \quad b=0$ and $a= \pm b$.

1) First subfamily of the second family of solutions; case: $a_{5}=a_{3}=0$, $v=a_{1}$ and $b \neq 0$

Equation (68) gives

$$
a^{2}-b^{2}=2 \alpha^{2} \sqrt[3]{90 \frac{a_{6}}{b_{3}}}, a_{6} b_{3} \succ 0 .
$$

Equations (70) and (72), by taking into account Equation (78), give, respectively

$$
b= \pm \sqrt{-\frac{b_{2}}{3 b_{3}}-\frac{70 \alpha^{2} a_{6}}{3 \sqrt[3]{300 a_{6} b_{3}^{2}}}-\frac{2 a_{4}}{3 \sqrt[3]{300 a_{6} b_{3}^{2}}}}, a_{6} \succ 0
$$


and

$$
\sqrt[3]{90 \frac{a_{6}}{b_{3}}}\left(b_{1}+2 b_{2} b^{2}+3 b_{3} b^{4}\right)-91 \alpha^{4} a_{6}-10 \alpha^{2} a_{4}-a_{2}=0 .
$$

From Equation (74), we obtain

$$
\omega=\left(b_{1}+b_{2} b^{2}+b_{3} b^{4}\right) b^{2}+\alpha^{6} a_{6}+\alpha^{4} a_{4}+\alpha^{2} a_{2},
$$

and next, Equation (78) gives

$$
a= \pm \sqrt{b^{2}+2 \alpha^{2} \sqrt[3]{90 \frac{a_{6}}{b_{3}}}}
$$

where $b$ is given by Equation (79) with $\operatorname{sign}\left(b_{3}\right)=\operatorname{sign}\left(a_{6}\right) \succ 0$. By pursuing, the combination of Equation (80) and Equation (81) leads to

$$
\omega=\left(\frac{91 \alpha^{4} a_{6}+\alpha^{2} a_{4}+a_{2}}{\sqrt[3]{90 \frac{a_{6}}{b_{3}}}}-b_{2} b^{2}-2 b_{3} b^{4}\right) b^{2}+\alpha^{6} a_{6}+\alpha^{4} a_{4}+\alpha^{2} a_{2},
$$

where $b$ is given by Equation (79). Finally, the first subfamily of the second family of solutions 1 is expresses as

$$
\begin{aligned}
q(x, t) & =\left[ \pm \sqrt{b^{2}+2 \alpha^{2} \sqrt[3]{90 \frac{a_{6}}{b_{3}}}} J_{1,0}(\alpha \xi)\right. \\
& \left. \pm i \sqrt{-\frac{b_{2}}{3 b_{3}}-\frac{70 \alpha^{2} a_{6}}{3 \sqrt[3]{300 a_{6} b_{3}^{2}}}-\frac{2 a_{4}}{3 \sqrt[3]{300 a_{6} b_{3}^{2}}}} J_{1,1}(\alpha \xi)\right] \mathrm{e}^{i \omega t},
\end{aligned}
$$

where $b$ is given by Equation (79) with $\operatorname{sign}\left(b_{3}\right)=\operatorname{sign}\left(a_{6}\right) \succ 0 ; a_{6} b_{3} \neq 0$ and the constraint given by Equation (83). This solution has a hybrid character which, according to the values of the coefficients $a, b, v, \alpha, \omega$ of the wave and those of parameters $a_{k}, k \in\{1 ; 2 ; 3 ; 4 ; 5 ; 6\}$ and $b_{r}, r \in\{1 ; 2 ; 3\}$ of the system generates either a bright, a kink or a combined solution of the two types which produces the hybrid solution. And the condition of existence of the solution given by Equation (84) is that: $\operatorname{sign}\left(b_{3}\right)=\operatorname{sign}\left(a_{6}\right) \succ 0$.

2) Second subfamily of the second family of solutions; case: $a_{5} \neq 0$, $a_{3} \neq 0, \quad v \neq a_{1}$ and $b=0$

For $b=0$, Equations (69), (71) and (73) are verified, and Equations (68), (70), (72), (74) become, respectively

$$
\begin{gathered}
b_{3} a^{6}-720 \alpha^{6} a_{6}=0, \\
b_{2} a^{4}+840 \alpha^{6} a_{6}+24 \alpha^{4} a_{4}=0, \\
b_{1} a^{2}-182 \alpha^{6} a_{6}-20 \alpha^{4} a_{4}-2 \alpha^{2} a_{2}=0, \\
\omega=\alpha^{6} a_{6}+\alpha^{4} a_{4}+\alpha^{2} a_{2} .
\end{gathered}
$$

Equation (87) gives 


$$
a= \pm \sqrt{182 \alpha^{6} \frac{a_{6}}{b_{1}}+20 \alpha^{2} \frac{a_{4}}{b_{1}}+2 \alpha^{2} \frac{a_{2}}{b_{1}}} .
$$

The combination of Equations (85) and (86) leads to the following relation

$$
a^{2}=-\frac{30 \alpha^{2} a_{6}}{b_{2}} 35 \alpha^{2} a_{6} b_{3}+a_{4} b_{3} ; b_{2} \neq 0 \text {. }
$$

From Equation (89) and Equation (90), when setting, $a^{2}=a^{2}$, we obtain the constraint below

$$
3185 \alpha^{6} a_{6}^{2} b_{3}+441 \alpha^{4} a_{6} a_{4} b_{3}+\alpha^{2}\left(35 a_{6} a_{2} b_{3}+10 a_{4}^{2} b_{3}\right)+15 a_{6} a_{2} b_{1}+a_{4} a_{2} b_{3}=0 .
$$

So, we obtain the second subfamily of the second family of solutions 1 as follows

$$
q(x, t)=\left[ \pm \sqrt{182 \alpha^{6} \frac{a_{6}}{b_{1}}+20 \alpha^{2} \frac{a_{4}}{b_{1}}+2 \alpha^{2} \frac{a_{2}}{b_{1}}} J_{1,0}(\alpha \xi)\right] \mathrm{e}^{i \omega t},
$$

with $b_{1} \neq 0, \omega=\alpha^{6} a_{6}+\alpha^{4} a_{4}+\alpha^{2} a_{2}$ and the constraint is given by Equation (91). Equation (92) shows that Equation (1) has the bright soliton as solution, the amplitude of which varies as the inverse of the square root of the coefficient $b_{1}$ of the cubic law.

3) Third subfamily of the second family of solutions; case: $a= \pm b$

This third subfamily of the second family of solutions of Equation (1) contains two sub-subfamilies, namely for cases: $a= \pm b=\beta ; a_{3} \neq a_{5} \neq 0 ; v \neq a_{1}$ and $a= \pm b=\mu, a_{3}=a_{5}=0, v=a_{1}$.

a) First sub-subfamily of the third subfamily of the second family of solutions; case: $a= \pm b=\beta ; \beta \in \mathfrak{R}^{*} ; a_{3} \neq a_{5} \neq 0 ; \quad v \neq a_{1}$

In this first case, if we are situated with respect to the conditions $a= \pm b=\beta$; $\beta \in \mathfrak{R}^{*} ; a_{3} \neq a_{5} \neq 0$ and $v \neq a_{1}$, we obtain within the framework of solving Equations (70), (71), (72), (73) and (75), the first sub-subfamily of the third subfamily of the second family of solutions as being

$$
q(x, t)=\left[\beta J_{1,0}(\alpha \xi) \pm i \beta J_{1,1}(\alpha \xi)\right] \mathrm{e}^{i \omega t},
$$

with $\omega=b_{1} b^{2}+b_{2} b^{4}+b_{3} b^{6}+225 \alpha^{6} a_{6} \quad ; \quad v=a_{1}-64 \alpha^{4} a_{5} \quad ; \quad a_{2}=259 \alpha^{4} a_{6}$; $a_{3}=-20 \alpha^{2} a_{5}$ and $a_{4}=-35 \alpha^{2} a_{6}$. Let us note here that, Equation (93) is an hybrid prototype which translates particular interactions between the two main types of bright and kink solitons, respectively. This interaction is revealed by the sequences $\left(a^{2}-b^{2}\right)^{k} b_{k}$, where $k \in\{1 ; 2 ; 3\}$, and which can be observed at the level of Equations (68), (70) and (72) and which in this case have imposed the condition $a= \pm b$. This result also shows that, for a given value of the intermodal dispersion coefficient, the wave speed is a linear function of the dispersion coefficient of order five, and implicitly, a linear function of the dispersion coefficient of order three $\left(a_{3}=-20 \alpha^{2} a_{5}\right)$.

b) Second sub-subfamily of the third subfamily of the second family of solutions; case: $a= \pm b=\mu ; \mu \in \mathfrak{R}^{*} ; a_{3}=a_{5}=0 ; \quad v=a_{1}$

In this second case, depending on the conditions $a= \pm b=\mu ; \mu \in \mathfrak{R}^{*}$; 
$a_{3}=a_{5}=0$ and $v=a_{1}$, the resolution of Equations (70), (71), (72), (73) and (75), leads to the second sub-subfamily of the third subfamily of the second family of solutions under the form

$$
q(x, t)=\left[\mu J_{1,0}(\alpha \xi) \pm i \mu J_{1,1}(\alpha \xi)\right] \mathrm{e}^{i \omega t},
$$

with $a_{4}=-35 \alpha^{2} a_{6} ; a_{2}=259 \alpha^{4} a_{6}$ and $\omega=b_{1} b^{2}+b_{2} b^{4}+b_{3} b^{6}+225 \alpha^{6} a_{6}$. Equation (94) is a hybrid prototype which also translates particular interactions between the two main types of bright and kink solitons, respectively. This interaction is revealed by the sequences $\left(a^{2}-b^{2}\right)^{k} b_{k}$, where $k \in\{1 ; 2 ; 3\}$, and which can be observed at the level of Equations (68), (70) and (72) and which in this case have imposed the condition $a= \pm b$, and consequently, leads to the following constraints $a_{3}=a_{5}=0$ and $v=a_{1}$.

\subsection{Graphical Verification of the Hybrid Trait of the Obtained Solutions}

This section getting charge to unveil the real nature of certain structures which are concealed behind the obtained analytical solutions and given by Equations (48), (57) and (65), to name just a few. Next, a detailed discussion is conducted in order to better compare these obtained structures with the theoretical forecasts. Five figures are presented, each displaying the different obtained structures in three dimensions. The graphical tool used here is the MAPLE.

From the above graphical representations, it clearly emerges that most of the obtained structures confirm of the analytical forecasts on the nature of the obtained solutions: these are hybrid dispersive optical solitons which will be the subject of discussions.

\section{Discussions}

From the previous study, it should be emphasized here that Equation (13) is a complex hybrid prototype of dispersive optical solitons which seems to harbor within it particular interactions between terms taken two by two of the same odd power $m=1 ; 3$ and contained in the different sub-packages (bright and dark mentioned above), and directly related to the cubic-quintic-septic law. These observations are reinforced by the appearance in the range Equations (15) to (29) of the expressions $\left(a^{2}-b^{2}\right)^{n} b_{n}$ and $\left(c^{2}-d^{2}\right)^{n} b_{n}, n \in\{1 ; 2 ; 3\}$ and whose extensions to the order $t$ in each case can be written respectively

$$
\left(\beta_{1}^{2}-\gamma_{1}^{2}\right) b_{1} ;\left(\beta_{1}^{2}-\gamma_{1}^{2}\right)^{2} b_{2} ;\left(\beta_{1}^{2}-\gamma_{1}^{2}\right)^{3} b_{3} ; \cdots ;\left(\beta_{1}^{2}-\gamma_{1}^{2}\right)^{t-1} b_{t-1} ;\left(\beta_{1}^{2}-\gamma_{1}^{2}\right)^{t} b_{t}
$$

and

$$
\left(\beta_{3}^{2}-\gamma_{3}^{2}\right) b_{1} ;\left(\beta_{3}^{2}-\gamma_{3}^{2}\right)^{2} b_{2} ;\left(\beta_{3}^{2}-\gamma_{3}^{2}\right)^{3} b_{3} ; \cdots ;\left(\beta_{3}^{2}-\gamma_{3}^{2}\right)^{t-1} b_{t-1} ;\left(\beta_{3}^{2}-\gamma_{3}^{2}\right)^{t} b_{t},
$$

where we have imagined an emergence at an order $j$ of the terms $\left(c^{2}-d^{2}\right)^{j} b_{j}$ and $\left(a^{2}-b^{2}\right)^{j} b_{j}, j \in\{1 ; 2 ; 3\}$ consequence of the initial and arbitrary choice of the ansatz (13) under the form 


$$
\psi(\xi)=\sum_{s=0}^{k}\left[\beta_{2 s+1} J_{2 s+1,0}(\alpha \xi)+i \gamma_{2 s+1} J_{2 s+1,2 s+1}(\alpha \xi)\right],
$$

It is important to note here that, Equations (95) and (96) are sequences which emerge in the range Equations (15) to (29) for values $s=0$ and $s=1$ introduced into Equation (97), respectively, when choosing the ansatz, with $a=\beta_{1}, b=\gamma_{1}, c=\beta_{3}$ and $d=\gamma_{3}$. This observation is undoubtedly necessary because it informs us a priori of what could be the major terms of the range equations and to move us away from certain errors during analytical calculations. Equation (95) highlights the interaction between sech and tanh while Equation (96) translates the interaction between the terms $\operatorname{sech}^{3}$ and $\tanh ^{3}$ of Equation (13) and which have favored the appearance of the cubic-quintic-septic law because equality $c=d$ prevents us from taking $b_{1}=0, b_{2}=0$, and $b_{3}=0$ (see Equations (15), (18) and (21)). The solutions found in the first family of solutions owe their existence to the appearance in the system of the Kerr law nonlinearity only(because $\left.b_{2}=b_{3}=0\right)$. On the other hand, the second family of solutions resulting from the condition $c=d=0$ owe its existence from the appearance in the propagation medium of the cubic-quintic-septic law (see Equations (68), (70) and (72)). Then, another peculiarity of the obtained dispersive optical solitons resides in their capacities of modulation following to the form and which making of them new hybrid prototypes which we estimate capable of changing form according to the difficulties encountered in their multiple mediums of propagation. This variation following to the form follows to the possibility of alternating the values of the coefficients $a, b, c, d$ and as well as the values of the parameters $\alpha$ and $v$ of the wave. This can be verified by paying close attention to the different obtained profiles for this purpose. Thereby:

Figure 1 introduces a set of four soliton profiles evolving from the doublebright given by Figure 1(a) to the bright given by Figure 1(d) through the intermediate forms given by Figure 1(b) and Figure 1(c) respectively. This figure reveals that, when we set the coefficients $b=0.9 ; c=d=-0.9$ and the parameters $\alpha=0.02 ; v=0.0012$, assigning values 0.9 in turn; $0.79 ; 0.59$ and 0.49 at coefficient a, Equation (48) is a hybrid prototype which generates multi-form structures belonging to the large bright family mainly.

Figure 2 is obtained by fixing the values of the coefficients $a=-0.3$ (with -0.49 as the last value); $b=-0.5 ; c=0$ and successively assigning to the coefficient $d$ the values $0.2 ; 0.13 ; 0.6$ and 0.15 . This exercise revealed the hybrid nature of the solution given by Equation (65). It offers four structures with a strong tendency to a bright type soliton. This can be elucidated by Figure 2(d) which is a bright which presents a flat top and reinforced by Figure 2(a) and Figure 2(c) like profiles with strong tendency bright.

Figure 3 displays a content made up of a group of four hybrid structures with a strong dark tendency. This trend can be confirmed by the profiles named Figure 3(a), Figure 3(c) (dark with a flat bottom) and Figure 3(d), against a bright represented by profile Figure $3(\mathrm{~b})$. To constitute this group and knowing that 
$c=0$, we successively gave the values $-0.3 ; 0.3 ; 0.09$ and -0.09 at coefficient $a$ while coefficient $b$ scans the values $0.05 ;-0.05 ; 0.002$ and 0.8 . The coefficient $d$ meanwhile, took the values $0.5 ; 0.2 ; 1$ and -1 . Here, $v=0.0012$ and $\alpha=0.02$.

Following the previous scenery, we estimate that such results will have a very great advantage during the propagation tests in laboratories where the researchers will be able to choose the form of the signal which they will want to inject into the system which they study quite simply by operating an appropriate choice of the values of these coefficients $a, b, c, d, \alpha$ and $v$ of the wave. In addition to all that has just been said, we believe that some of these new prototypes of hybrid dispersive optical solitons may find their applications in nonlinear fiber optics when the refractive index of light is proportional to the intensity; others in neuroscience where optical solitons have been spotted [44] [45] [46] and in the fluid media, more precisely in the context of waves in deep waters, etc. And thereafter, these solutions may also make it possible to detect new behaviors in the propagation media in which the Cubic-Quintic-Septic law appears, and thus contributes to the progress of technologies of the information. At the end of the discussions, it should be noted that most of the obtained results are different from those proposed in [5] [6] [7] [11] [17] [18] [19] [20] [26] [27] [28] [29] [47]. This difference can be observed at two levels:

- first from the analytical point of view by its mathematical form given by Equation (13). It is a package [25] [30] [31] that contains within its bosom four terms of which the first and third terms are representative of the bright type soliton, the second is a kink while the fourth term is a double-kink [34].
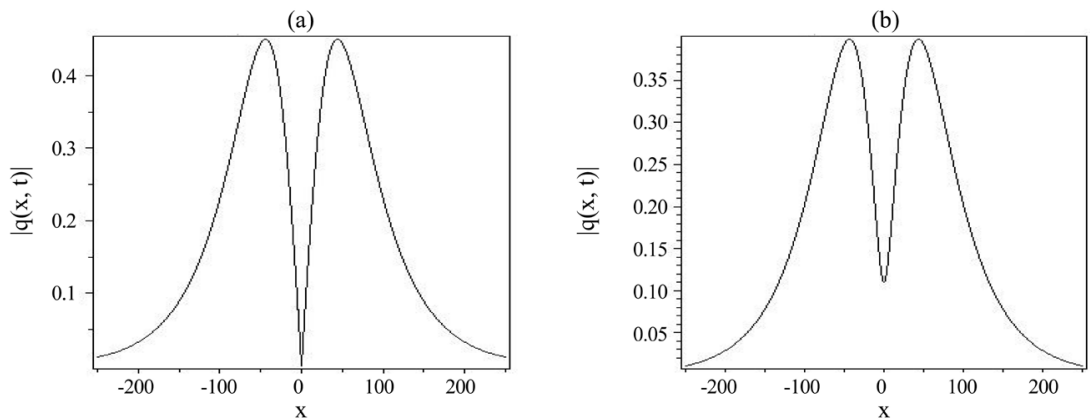

(c)
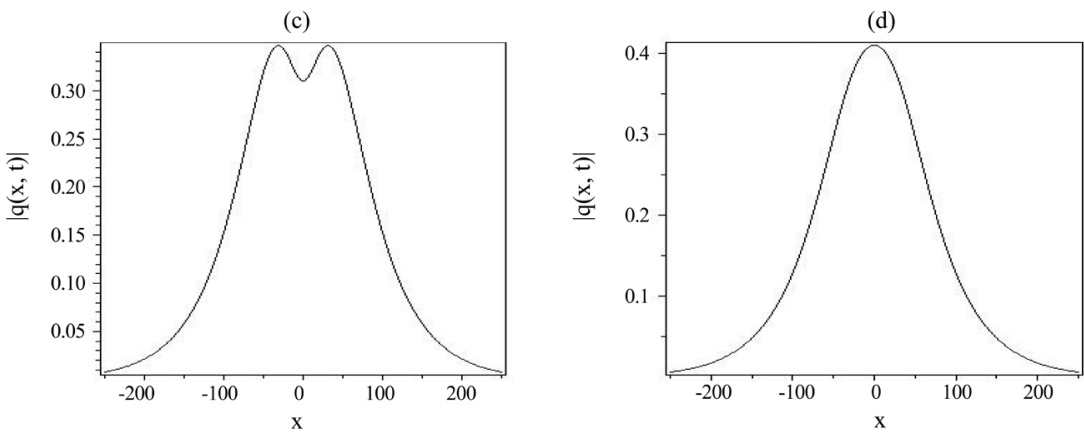

Figure 1. Graphical representation given by Equation (48) for $\alpha=0.02 ; v=0.0012$ : Hybrid-bright dispersive optical soliton: (a) $a=0.9 ; b=0.9 ; c=-0.9 ; d=-0.9$; (b) $a=0.79 ; b=0.9 ; c=-0.9 ; d=-0.9$; (c) $a=0.59 ; b=0.9 ; c=-0.9 ; d=-0.9$; (d) $a=0.49 ; b=0.9 ; c=-0.9 ; d=-0.9$. 


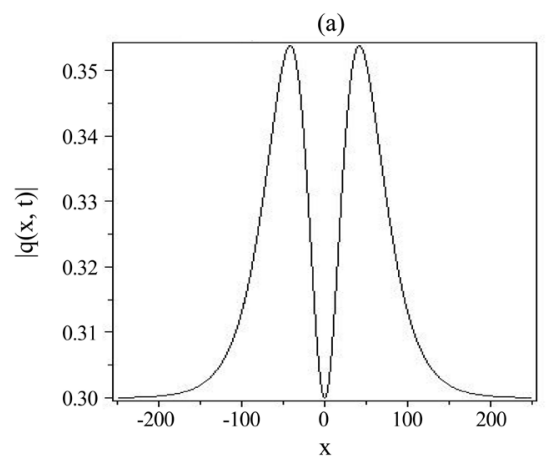

(c)

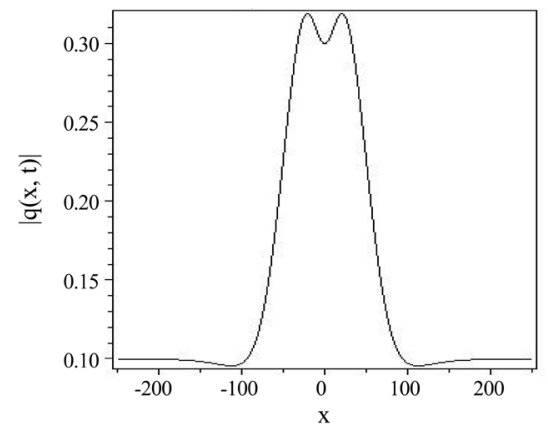

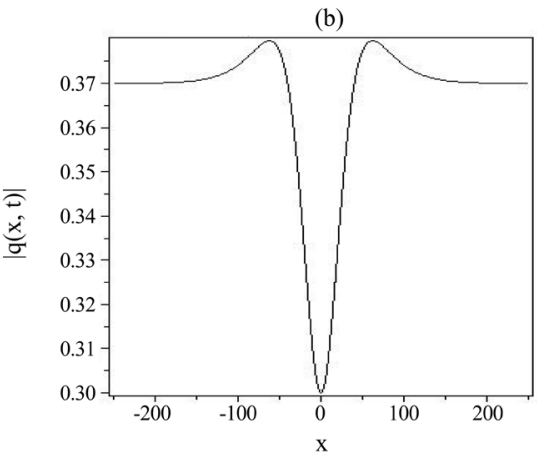

(d)

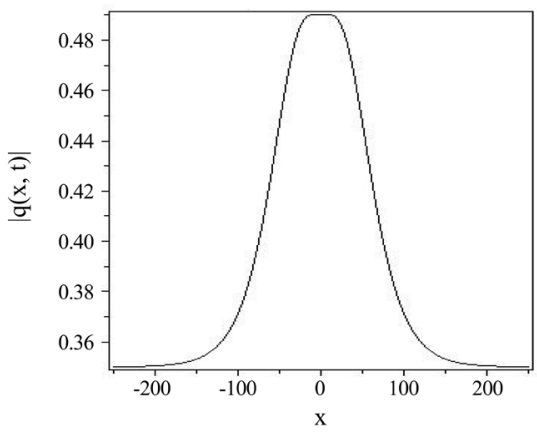

Figure 2. Graphical representation given by Equation (65) for $\alpha=0.02 ; v=0.0012$ : Hybrid bright-dark dispersive optical soliton: (a) $a=-0.3 ; b=-0.5 ; c=0 ; d=0.2$; (b) $a=-0.3 ; b=-0.5 ; c=0 ; d=0.13 ;$ (c) $a=-0.3 ; b=-0.5 ; c=0 ; d=0.6$ (d) $a=-0.49 ; b=-0.5 ; c=0 ; d=0.15$.

(a)

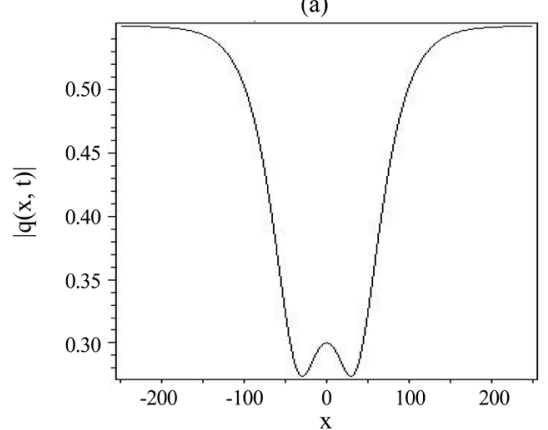

(c)

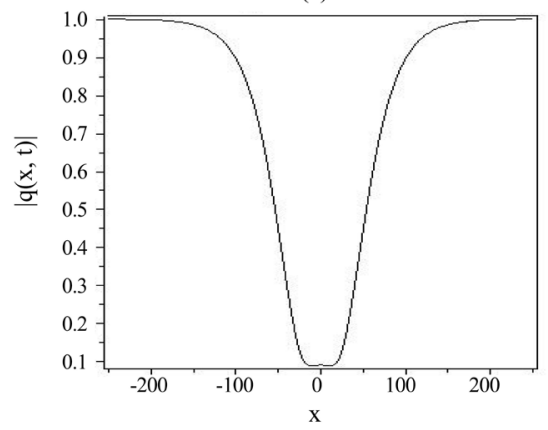

(b)

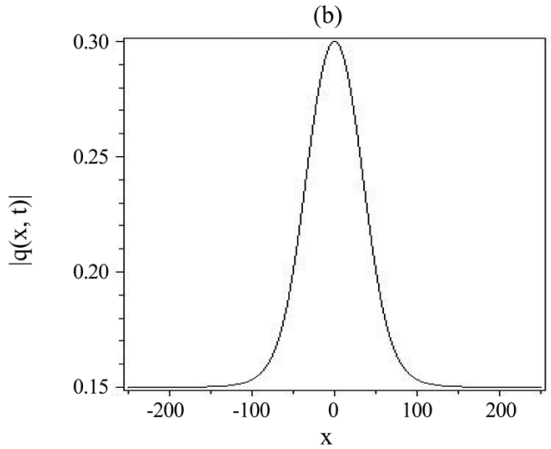

(d)

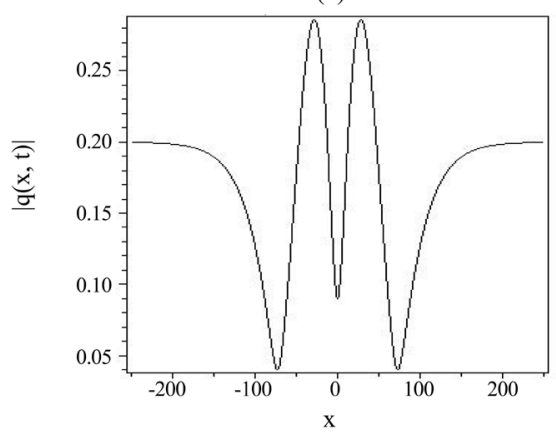

Figure 3. Graphical representation given by Equation (65) for $\alpha=0.02 ; v=0.0012$ : Hybrid dark-bright dispersive optical soliton: (a) $a=-0.3 ; b=0.05 ; c=0 ; d=0.5$; (b) $a=0.3 ; b=-0.05 ; c=0 ; d=0.2 ;$ (c) $a=0.09 ; b=0.002 ; c=0 ; d=1$ (d) $a=-0.09 ; b=0.8 ; c=0 ; d=-1$. 
- then, from a numerical point of view, for example, Figure 3(d) displays a hybrid or multiform wave structure which reveals the bright and dark types that are qualified as double dark-double bright solitary wave.

Whereas, in [7] and [18], the authors proposed exact bright, dark solitons and the singular optical solitons while in [17], authors proposed the highly dispersive singular optical solitons.

\section{Conclusion}

Ultimately, it should be pointed out that, thanks to the BDKm theory, we were able to locate and unearth new hybrid prototypes of dispersive optical solitons as much on their mathematical forms as on their profiles. This method has revealed a certain affinity coupled (between certain terms of the ansatz (13)) to being regroup in a sorts of power of the difference of two squares of exponent $n$, engaging their amplitudes and in link to cubic-quintic-septic law, and occurring in the forms $\left(c^{2}-d^{2}\right)^{n} b_{n}$ and $\left(a^{2}-b^{2}\right)^{n} b_{n}, n \in\{1 ; 2 ; 3\}$ respectively. From this affinity it emerges that some of these solutions owe their existence only to the appearance in the system of non-linearity of Kerr type through the sequence $\left(c^{2}-d^{2}\right) b_{1}$ (see the first term of Equation (43)) and which gave rise to the condition $c= \pm d$. And also of the appearance of the cubic-quintic-septic law through the sequence $\left(a^{2}-b^{2}\right)^{n} b_{n}, n \in\{1 ; 2 ; 3\} \quad$ (see Equations (68), (70), (72)) and which gave rise to the condition $a= \pm b$ and concerns the second family of solutions (case $c=0, d=0$ ). The profiles displayed by Figures 1-3 confirmed the hybrid characters of the obtained dispersive optical solitons. At the same time, these Figures indicate that a precise choice can be made in advance on the profiles of the hybrid dispersive solitons that one would like to obtain by simply making an appropriate choice of the values of the coefficients $a, b, c, d$ and those of parameters $v$ and $\alpha$ of the initial wave given by Equation (13). Thus, as the form of the signal which one wants to obtain varies according to the values of the real constants $a, b, c, d, v$ and $\alpha$, that supposes that one can control the energies of the nonlinear systems whose the dynamics are governed by the Schrödinger Equation (1) by simply playing on the values of these real constants. These new hybrid prototypes of dispersive optical solitons translate the new behaviors that can be developed by systems whose dynamics are described by Equation (1). Since the transmission of data through optical fibers is the responsibility of optical solitons [2] [3] [6] [26] [27] [28] [29], we believe that the new hybrid prototypes of dispersive optical solitons that we have proposed in the framework of this work will be able to respond to the requirement in recent years, of information technology which is, the improvement of optical fiber transmission systems associated with the extraction of optical solitons. We believe that a good understanding of the hybrid dispersive optical solitons highlighted in the context of this work may also allow to grasp the physical description of systems whose dynamics are governed by Equation (1) in order to provide a relevant improvement of complex problems. However, a continuous ex- 
ploration deserves to be maintained in this direction in order to further enrich the literatures of new types of dispersive optical soliton type structures whose needs are no longer in doubt.

\section{Acknowledgements}

Both Ministries of Higher Education of Cameroon and Gabon through their programs of support to research have enabled us to carry out this work, that they receive here our sincere thanks. The authors also thank support from Keutcha Padji Aurélien which, despite his manifold occupations, he never stopped reading in order to improve the quality of English as presented in this manuscript.

\section{Conflicts of Interest}

The authors declare no conflicts of interest regarding the publication of this paper.

\section{References}

[1] Hirota, R. (1971) Exact Solution of the Korteweg-de Vries Equation for Multiple Collisions of Solitons. Physical Review Letters, 27, 1192-1194. https://doi.org/10.1103/PhysRevLett.27.1192

[2] Lee, J.-H., Lin, C.-K. and Pashaev, O.K. (2004) Shock Waves, Chiral Solitons and Semiclassical Limit of One-Dimensional Anyons. Chaos, Solitons and Fractals, 19, 109-128. https://doi.org/10.1016/S0960-0779(03)00084-5

[3] Tariq, K.U., Younis, M., Rezazadeh, H., Rizvi, S.T.R. and Osman, M.S. (2018) Optical Solitons with Quadratic-Cubic Nonlinearity and Fractional Temporal Evolution. Modern Physics Letters B, 32, 1850317-185049. https://doi.org/10.1142/S0217984918503177

[4] Seadawy, A.R., Arshad, M. and Lu, D. (2017) Stability Analysis of New Exact Traveling-Wave Solutions of New Coupled KdV and New Coupled Zakharov-Kuznetsov Systems. European Physical Journal Plus, 132, 162-180. https://doi.org/10.1140/epjp/i2017-11437-5

[5] Ngouo Tchinda, C. and Bogning, J.R. (2020) Solitary Waves and Property Management of Nonlinear Dispersive and Flattened Optical Fiber. American Journal Optics and Photonics, 8, 27-32. https://doi.org/10.11648/j.ajop.20200801.13

[6] Mahak, N. and Akram, G. (2019) Novel Approaches to Extract Soliton Solutions of the $(1+1)$ Dimensional Fokas-Lenells Equation by Means of the Complex Transformation. Optik, 192, 162912-162919. https://doi.org/10.1016/j.ijleo.2019.06.012

[7] Bhrawy, A.H., Alshaery, A.A., Hilal, E.M., Manrakhan, W.N., Savescu, M. and Biswas, A. (2014) Dispersive Optical Solitons with Schrödinger-Hirota Equation. Journal of Nonlinear Optical Physics \& Materials, 23, 1450014-1450034. https://doi.org/10.1142/S0218863514500143

[8] Ebaid, A., El-Zahar, E.R., Aljohani, A.F., Salah, B., Krid, M. and Machado, J.T. (2019) Exact Solutions of the Generalized Nonlinear Fokas-Lennells Equation. Results in Physics, 14, 102472-102476. https://doi.org/10.1016/j.rinp.2019.102472

[9] Osman, M.S., Lu, D. and Khater, M.M.A. (2019) A Study of Optical Wave Propagation in the Nonautonomous Schrödinger-Hirota Equation with Power-Law Nonlinearity. Results in Physics, 13, 102157-102160. 
https://doi.org/10.1016/j.rinp.2019.102157

[10] Biswas, A. (2004) Stochastic Perturbation of Optical Solitons in Schrödinger-Hirota Equation. Optics Communications, 239, 461-466.

https://doi.org/10.1016/j.optcom.2004.06.047

[11] Zhang, Z.-Y. (2015) Jacobi Elliptic Function Expansion Method for the Modified Korteweg-de Vries-Zakharov Kuznetsov and the Hirota Equations. Romanian Journal of Physics, 60, 1384-1394.

[12] Biswas, A., Yildrim, Y., Yasar, E., Zhou, Q., Alshomrani, A.S. and Belic, M. (2019) Optical Soliton Perturbation in Parabolic Law Medium Having Weak Non-Local Nonlinearity by a Couple of Strategic Integration Architectures. Results in Physics, 13, 102334-102346. https://doi.org/10.1016/j.rinp.2019.102334

[13] Abdou, M.A. and Elhanbaly, A. (2007) Construction of Periodic and Solitary Wave Solutions by the Extended Jacobi Elliptic Function Expansion Method. Communications in Nonlinear Science and Numerical Simulation, 12, 1229-1241. https://doi.org/10.1016/j.cnsns.2006.01.013

[14] Kudryashov, N.A. (2019) First Integrals and General Solution of the Traveling Wave Reduction for Schrödinger Equation with Anti-Cubic Nonlinearity. Optik, 185, 665-671. https://doi.org/10.1016/j.ijleo.2019.03.167

[15] Yildirim, Y. (2019) Optical Soliton Molecules of Manakov Model by Trial Equation Technique. Optik, 185, 1146-1151. https://doi.org/10.1016/j.ijleo.2019.04.041

[16] Biswas, A., Ekici, M., Sonmezoglu, A. and Belic, M.R. (2019) Highly Dispersive Optical Solitons with Cubic-Quintic-Septic Law by Extended Jacobi's Elliptic Function Expansion. Optik, 183, 571-578. https://doi.org/10.1016/j.ijleo.2019.02.127

[17] Biswas, A., Ekici, M., Sonmezoglu, A. and Belic, M.R. (2019) Highly Dispersive Optical Solitons with Cubic-Quintic-Septic Law by Exp-Expansion. Optik, 186, 321-325. https://doi.org/10.1016/j.ijleo.2019.04.085

[18] Biswas, A., Ekici, M., Sonmezoglu, A. and Belic, M.R. (2019) Highly Dispersive Optical Solitons with Cubic-Quintic-Septic Law by F-Expansion. Optik, 182, 897-906. https://doi.org/10.1016/j.ijleo.2019.01.058

[19] Biswas, A., Vega-Guzman, J., Mahmood, M.F., Khan, S., Ekici, M., Zhou, Q., Moshoko, S.P. and Belic, M.R. (2019) Highly Dispersive Optical Solitons with Undetermined Coefficients. Optik, 182, 890-896. https://doi.org/10.1016/j.ijleo.2019.01.087

[20] Ur Rehman, H., Ullah, N. and Imran, M.A. (2019) Highly Dispersive Optical Solitons Using Kudryashov's Method. Optik, 199, 163349-163355. https://doi.org/10.1016/j.ijleo.2019.163349

[21] Djeumen Tchaho, C.T., Bogning, J.R. and Kofané, T.C. (2010) Construction of the Analytical Solitary Wave Solutions of Modified Kuramoto-Sivashinsky Equation by the Method of Identification of Coefficients of the Hyperbolic Functions. Far East Journal of Dynamical Systems, 14, 17-34.

[22] Djeumen Tchaho, C.T., Bogning, J.R. and Kofané, T.C. (2011) Multi-Soliton Solutions of the Modified Kuramoto-Sivashinsky Equation by the BDK Method. Far East Journal of Dynamical Systems, 15, 83-98.

[23] Bogning, J.R., Djeumen Tchaho, C.T. and Kofané, T.C. (2012) Construction of the Soliton Solutions of the Ginzburg-Landau Equations by the New Bogning-Djeumen Tchaho-Kofané Method. Physica Scripta, 85, 25013-25017. https://doi.org/10.1088/0031-8949/85/02/025013

[24] Djeumen Tchaho, C.T. (2015) New Method of Construction of the Solitary Wave 
Solutions of Some Physical Nonlinear Partial Differential Equations Doctorat. Ph.D. Thesis, University of Yaounde I, Yaounde.

[25] Bogning, J.R. (2019) Mathematics for Nonlinear Physics: Solitary Waves in the Center of Resolutions of Dispersive Nonlinear Partial Differential Equations. Dorrance Publishing Co., Pittsburgh.

[26] Rezazadeh, H., Tariq, K.U., Sabi'u, J. and Bekir, A. (2020) Abundant Traveling Wave Solutions to the Resonant Nonlinear Schrödinger's Equation with Variable Coefficients. Modern Physics Letters B, 34, 2050118-2050128. https://doi.org/10.1142/S0217984920501183

[27] Inc, M., Aliyu, A.I. and Yusuf, A. (2017) Dark Optical, Singular Solitons and Conservation Laws to the Nonlinear Schrödinger's Equation with Spatio-Temporal Dispersion. Modern Physics Letters B, 31, Article ID: 1750163. https://doi.org/10.1142/S0217984917501639

[28] Das, A., Biswas, A., Ekici, M., Zhou, Q., Alshomrani, A.S. and Belic, M.R. (2019) Optical Solitons with Complex Ginzburg-Landau Equation for Two Nonlinear Forms Using F-Expansion. Chinese Journal of Physics, 61, 255-261. https://doi.org/10.1016/j.cjph.2019.08.009

[29] Biswas, A. (2003) Optical Solitons: Quasistationarity versus Lie Transform. Optical and Quantum Electronics, 35, 979-998. https://doi.org/10.1023/A:1025121931885

[30] Bogning, J.R. (2019) Mathematics for Nonlinear Physics: The Implicit Bogning Functions and Applications. Lambert Academic Publishing, Saarbrücken.

[31] Bogning, J.R. (2020) Elements of Analytical Mechanics and Quantum Physics. Lambert Academic Publishing, Saarbrücken.

[32] Djeumen Tchaho, C.T., Bogning, J.R. and Kofané, T.C. (2012) Modulated Soliton Solution of the Modified Kuramoto-Sivashinsky's Equation. American Journal of Computational and Applied Mathematics, 2, 218-224.

https://doi.org/10.5923/j.ajcam.20120205.03

[33] Bogning, J.R., Djeumen Tchaho, C.T. and Kofané, T.C. (2012) Generalization of the Bogning-Djeumen Tchaho-Kofané Method for the Construction of the Solitary Waves and the Survey of the Instabilities. Far East Journal of Dynamical Systems, 20, 101-119. https://doi.org/10.5923/j.ajcam.20120205.03

[34] Djeumen Tchaho, C.T., Omanda, H.M. and Belobo Belobo, D. (2018) Hybrid Solitary Waves for the Generalized Kuramoto-Sivashinsky Equation. The European Physical Journal Plus, 133, 387-395. https://doi.org/10.1140/epjp/i2018-12218-4

[35] Djeumen Tchaho, C.T., Omanda, H.M., N’tchayi Mbourou, G., Bogning, J.R. and Koafné, T.C. (2019) Multi-Form Solitary Waves Solutions of the KdV-Burgers-Kuramoto Equation. Journal of Physics Communications, 3, 105013-105023. https://doi.org/10.1088/2399-6528/ab4ba1

[36] Njikue, R., Bogning, J.R. and Kofané, T.C. (2018) Exact Bright and Dark Solitary Wave Solutions of the Generalized Higher-Order Nonlinear Schrödinger Equation Describing the Propagation of Ultra-Short Pulse in Optical Fibers. Journal of Physics Communications, 2, 25030-25039. https://doi.org/10.1088/2399-6528/aaaf3b

[37] Bogning, J.R. (2018) Exact Solitary Wave Solutions of the (3+1)-Modified B-Type Kadomtsev-Petviashvili Family Equations. American Journal of Computational and Applied Mathematics, 8, 85-92.

[38] Bogning, J.R., Djeumen Tchaho, C.T. and Omanda, H.M. (2016) Combined Solitary Wave Solutions in Higher-order Effects Optical Fibers. British Journal of Mathematics \& Computer Science, 13, 1-12. https://doi.org/10.9734/BJMCS/2016/10620

[39] Bogning, J.R., Fautso Kuiaté, G., Omanda, H.M. and Djeumen Tchaho, C.T. (2015) 
Combined Peakons and Multiple-Peak Solutions of the Camassa-Holm and Modified KdV Equations and Their Conditions of Obtention. Physics Journal, 1, 367-374.

[40] Bogning, J.R., Porsezian, K., Fautso Kuiaté, G. and Omanda, H.M. (2015) Gap Solitary Pulses Induced by the Modulational Instability and Discrete Effects in Array of Inhomogeneous Optical Fibers. Physics Journal, 1, 216-224.

[41] Tiague Takongmo, G. and Bogning, J.R. (2018) Construction of Solutions in the Shape (Pulse; Pulse) and (Kink; Kink) of a Set of Two Equations Modeled in a Nonlinear Inductive Electrical Line with Crosslink Capacitor. American Journal of Cir cuits, Systems and Signal Processing, 4, 28-35.

[42] Tiague Takongmo, G. and Bogning, J.R. (2018) Construction of Breather Soliton Solutions of a Modeled Equation in a Discrete Nonlinear Electrical Line and the Survey of Modulationnal Instability. Journal of Physics Communications, 2, 115007-115019. https://doi.org/10.1088/2399-6528/aaeaal

[43] Tiague Takongmo, G. and Bogning, J.R. (2018) Coupled Soliton Solutions of Modeled Equations in a Noguchi Electrical Line with Crosslink Capacitor. Journal of Physics Communications, 2, 105016-105026. https://doi.org/10.1088/2399-6528/aae7e6

[44] Biswas, A., Milovic, D. and Milic, D. (2011) Solitons in Alpha-Helix Proteins by He's Variational Principle. International Journal of Biomathematics, 4, 423-429. https://doi.org/10.1142/S1793524511001325

[45] Biswas, A., Kara, A.H., Savscu, M., Bokhari, A.H. and Zaman, F.D. (2013) Solitons and Conservation Laws in Neurosciences. International Journal of Biomathematics, 6, Article ID: 1350017. https://doi.org/10.1142/S1793524513500174

[46] Biswas, A., Milovic, D., Savescu, M., Mahmood, M.F., Khan, K.R. and Kohl, R. (2012) Optical Soliton Perturbation in Nanofibers with Improved Nonlinear Schrödinger's Equation by Semi-Inverse Variational Principle. Journal of Nonlinear Optical Physics \& Materials, 21, Article ID: 1250054. https://doi.org/10.1142/S0218863512500543

[47] Kudryashov, A. (2020) Highly Dispersive Solitary Wave Solutions of Perturbed Nonlinear Schrödinger Equations. Applied Mathematics and Computation, 371, Article ID: 124972. https://doi.org/10.1016/j.amc.2019.124972 